\title{
Ultrafast Charge and Triplet State Formation in Diketopyrrolopyrrole Low Band Gap Polymer/Fullerene Blends: Influence of Nanoscale Morphology of Organic Photovoltaic Materials on Charge Recombination to the Triplet State
}

\author{
René M. Williams, ${ }^{1}$ Hung-Cheng Chen, ${ }^{1}$ Daniele Di Nuzzo, ${ }^{2}$ Stephan C. J. Meskers, ${ }^{2}$ and \\ René A. J. Janssen ${ }^{2}$ \\ ${ }^{1}$ Molecular Photonics Group, Van't Hoff Institute for Molecular Sciences (HIMS), Universiteit van Amsterdam, Science Park 904, \\ 1098 XH Amsterdam, Netherlands \\ ${ }^{2}$ Molecular Materials and Nanosystems, Eindhoven University of Technology, P.O. Box 513, 5600 MB Eindhoven, Netherlands
}

Correspondence should be addressed to René M. Williams; r.m.williams@uva.nl and René A. J. Janssen; r.a.j.janssen@tue.nl

Received 19 October 2016; Accepted 25 December 2016; Published 13 April 2017

Academic Editor: Jau-Wern Chiou

Copyright (C) 2017 René M. Williams et al. This is an open access article distributed under the Creative Commons Attribution License, which permits unrestricted use, distribution, and reproduction in any medium, provided the original work is properly cited.

\begin{abstract}
Femtosecond transient absorption spectroscopy of thin films of two types of morphologies of diketopyrrolopyrrole low band gap polymer/fullerene-adduct blends is presented and indicates triplet state formation by charge recombination, an important loss channel in organic photovoltaic materials. At low laser fluence (approaching solar intensity) charge formation characterized by a $1350 \mathrm{~nm}$ band (in $250 \mathrm{fs}$ ) dominates in the two PDPP-PCBM blends with different nanoscale morphologies and these charges recombine to form a local polymer-based triplet state on the sub-ns timescale (in $\sim 300$ and $\sim 900 \mathrm{ps}$ ) indicated by an $1100 \mathrm{~nm}$ absorption band. The rate of triplet state formation is influenced by the morphology. The slower rate of charge recombination to the triplet state (in $\sim 900 \mathrm{ps}$ ) belongs to a morphology that results in a higher power conversion efficiency in the corresponding device. Nanoscale morphology not only influences interfacial area and conduction of holes and electrons but also influences the mechanism of intersystem crossing (ISC). We present a model that correlates morphology to the exchange integral and fast and slow mechanisms for ISC (SOCT-ISC and H-HFI-ISC). For the pristine polymer, a flat and unstructured singlet-singlet absorption spectrum (between 900 and $1400 \mathrm{~nm}$ ) and a very minor triplet state formation (5\%) are observed at low laser fluence.
\end{abstract}

\section{Introduction}

Since the first observation of photo-induced electron transfer from a conducting polymer to buckminsterfullerene $\left(\mathrm{C}_{60}\right)$ in 1992 [1], crucial steps towards better materials for plastic solar cells have been made. The introduction of a more soluble fullerene adduct (PCBM) [2], the development of the bulk heterojunction concept [3], and the switch to thiophene-based polymers (P3HT) [4] were paramount. The next steps that have to be taken in order to improve these solar cells are the absorption of a larger part of the available solar radiation and minimizing charge loss [5]. Efficiently harvesting a larger part of the solar spectrum and controlling charge recombination while maintaining the right nanoscale morphology for efficient charge generation and optimizing molecular organization for charge transport are the current challenges in organic photovoltaic (OPV) research. Low band gap materials are therefore anticipated to be a key component in the next generations of organic solar cells [6-9]. With this type of material combined with [70]PCBM, up to $11.7 \%$ power conversion efficiency (PCE) has been obtained $[10,11]$.

The diketopyrrolopyrrole (DPP) unit has gained a lot of interest as a component for small molecule-based OPV and has also been incorporated into polymers for solar cells $[12,13]$. DPP-based polymer materials (see Figure 1) can 


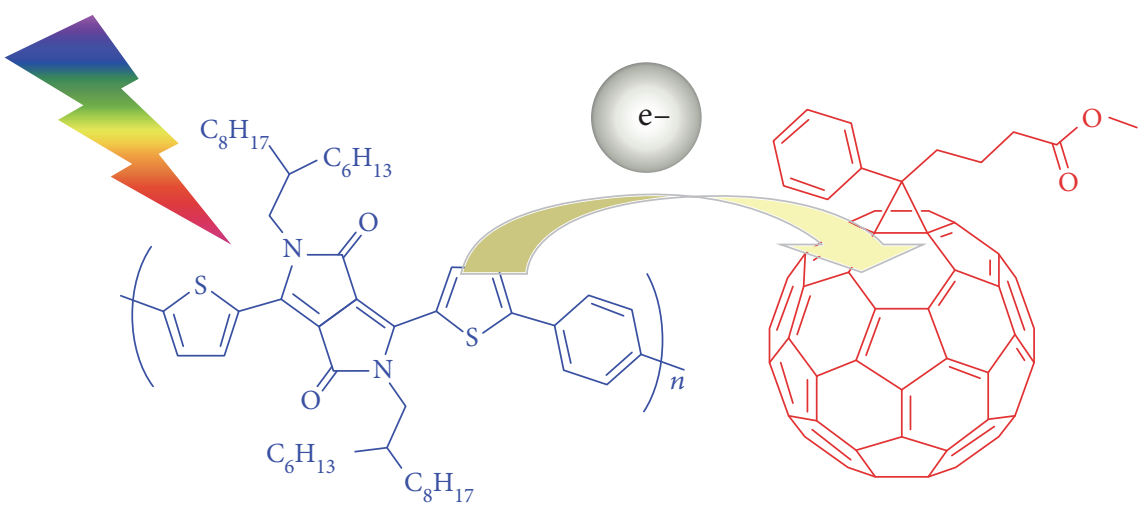

FIgURE 1: Molecular structures of the components used in this study, the PDPP polymer and [60]PCBM.

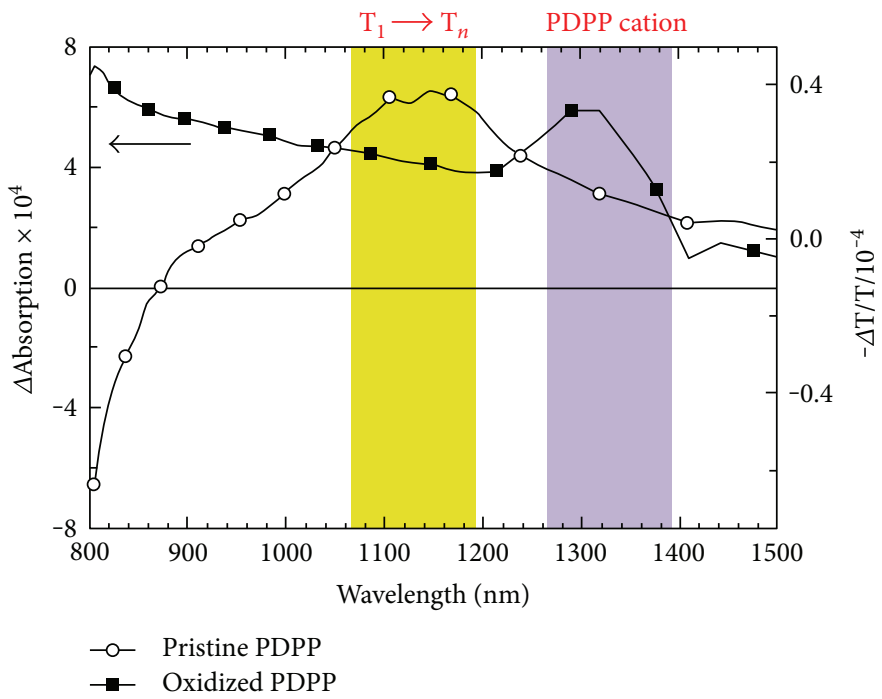

FIGURE 2: Triplet-triplet absorption and radical cation absorption of the PDPP polymer (converted to wavelength scale; data taken from [14]).

absorb sunlight up to $\sim 900 \mathrm{~nm}$ and it has been shown that these materials can yield OPV devices with a $\sim 5$ to $\sim 6 \%$ power conversion efficiency [14]. The use of a cosolvent like 1,8-diiodooctane (DIO) or ortho-dichlorobenzene (ODCB) during spin coating is crucial for obtaining the optimized nanostructure and correlated higher efficiency, mainly attributed to a better ordering in the fullerene phase. Optimized [60]PCBM devices were reported with a short circuit current $J_{\mathrm{SC}}=9.3 \mathrm{~mA} \mathrm{~cm}^{-2}$, an open circuit voltage $V_{\mathrm{OC}}=0.78 \mathrm{~V}$, a fill factor $\mathrm{FF}=0.63$, and a $\mathrm{PCE}=4.6 \%$. The HOMO and LUMO levels of the polymer were estimated to be -5.35 and $-3.53 \mathrm{eV}$ versus vacuum. The absorption onset of the films was reported to be $1.53 \mathrm{eV}$. The external quantum efficiencies ranged from $\mathrm{EQE}=45 \%(600-750 \mathrm{~nm}$ region $)$ to $20 \%$ at $530 \mathrm{~nm}$. Furthermore, steady-state photo-induced absorption spectroscopy showed the presence of positive polarons at $1350 \mathrm{~nm}$ and triplet states at $1100 \mathrm{~nm}$ (see Figure 2), the latter indicating triplet state formation via charge transfer states [14].
Thus, while a larger part of the solar spectrum can be harvested with these DPP-based devices (as compared to, e.g., those based on PCBM/P3HT that have their main P3HT absorption only until $675 \mathrm{~nm}$ ) [15], the output is not proportionally increased. Clearly, more loss channels are present that reduce the efficiency, and now, triplet state formation plays a role in the charge loss in these materials.

Charge loss can occur through trap states and by Langevin-type recombination at the interface of percolation pathways. It has recently been shown that, through spin statistics, triplet states can be formed through this recombination process on the sub-microsecond to millisecond timescale [16]. Sub-ns timescale charge loss through triplet recombination channels in polymer solar cell materials has also gained a lot of interest in the last years [17]. Furthermore, it is important to note that in a recent report, Howard et al. stated that currently there is no insight into the exact mechanism of charge recombination to the triplet state [18]. 
Here, we report on the primary photophysical events occurring in two types of thin-film blends containing a low band gap polymer (PDPP) and PCBM (see also Figure 1) as well as on the events occurring in the pristine polymer thin film as observed with femtosecond transient absorption spectroscopy (fs-TA). The official name of the PDPP polymer is poly[ $\{2,5$-bis(2-hexyldecyl)-2,3,5,6-tetrahydro-3, 6-dioxopyrrolo[3,4-c]pyrrole-1,4-diyl $\}$-alt- $\left\{\left[2,2^{\prime}\right.\right.$-(1,4-phenylene) bisthiophene]-5, $5^{\prime}$-diyl $\left.\}\right]$ ).

At low laser fluence, where singlet-singlet annihilation or processes like "hot singlet fission" [19] do not play a role, a normal excited singlet state is observed for the polymer film.

For the blends of PCBM and the low band gap polymer at low laser fluences (approaching solar intensity) ultra-fast charge generation dominates, but clearly triplet state formation occurs on a sub-ns timescale. Morphology influences charge generation and triplet formation. Fast charge recombination to the triplet state is thus identified as an important charge loss in these blends. The potential gain in efficiency due to the low band gap is counter effected by the lower triplet state of the polymer, which is partly accessible from the charge transfer state. Our results indicate that the efficiency of the plastic solar cells made of these materials will be highly influenced by the solar-light intensity, and is expected to be relatively high at low solar fluence (comparing, e.g., 1 versus 10 sun). Extrapolation to the limit of zero laser fluence projects a (triplet) loss-corrected PCE of 9.6\% for these materials.

We present a model for the influence of nanoscale morphology on charge recombination to the triplet state. We use previous studies of molecular electron donor acceptor systems in solution regarding triplet state formation by charge recombination from compact charge transfer states. We combine this with recent results that suggest the occurrence of long range photo-induced electron transfer occurring in optimized photovoltaic blends, implying a large distance between hole and electron.

\section{Experimental}

2.1. Film Preparation. The synthetic procedures for the preparation of PDPPTPT have been reported [14]. [60]PCBM (purity $>99 \%$ ) was obtained from Solenne B.V. The photoactive layer was deposited on quartz by spin coating a chloroform solution containing $6 \mathrm{mg} \mathrm{mL} \mathrm{mL}^{-1}$ PDPPTPT and $12 \mathrm{mg} \mathrm{mL}^{-1} \mathrm{PCBM}$ and $25 \mathrm{mg}$ ortho-dichlorobenzene (or $25 \mathrm{mg}$ 1,8-diiodooctane) per $\mathrm{mL}$ solution. Thickness of the active layers $(80-90 \mathrm{~nm})$ was measured on a Veeco Dektak 150 profilometer. Optical densities at $530 \mathrm{~nm}$ were between 0.098 and 0.11 .

\subsection{Femtosecond Transient Absorption Spectroscopy. Femto-} second transient absorption experiments were performed with a Spectra-Physics Hurricane Titanium:Sapphire regenerative amplifier system. The full spectrum setup was based on an optical parametric amplifier (Spectra-Physics OPA $800 \mathrm{C}$ ) as the pump. The residual fundamental light, from the pump OPA, was used for NIR probe-light generation, using a sapphire plate. For femtosecond transient absorption in the NIR region, a Control Development NIR-256L-1.7T1-USB optical spectrometer system and an InGaAs detector with 512 element arrays responding to wavelengths ranging from 900 to $1500 \mathrm{~nm}$ were used. The polarization of the pump light was controlled by a Berek Polarization Compensator (New Focus). The Berek Polarizer was always included in the setup to provide the magic angle conditions. The probe light was double passed over a delay line (Physik Instrumente, M-531DD) that provides an experimental time window of $3.6 \mathrm{~ns}$ with a maximal resolution of $0.6 \mathrm{fs}$ per step. The OPA was used to generate excitation pulses at $530 \mathrm{~nm}$. The laser output was typically $28 \mu \mathrm{J} / \mathrm{cm}^{2}$ per pulse (130 fs FWHM) with a repetition rate of $1 \mathrm{kHz}$. Laser fluence was adjusted with a neutral density filter wheel. The solution samples were placed into cells of $2 \mathrm{~mm}$ path length (Hellma) and were stirred with a downward-projected PTFE shaft using a direct-drive spectro-stir (SPECTRO-CELL).

2.3. Global and Target Analysis Using Glotaran. The analysis of the time-resolved spectroscopic data consisting of a three-dimensional dataset (wavelength, time and absorption difference intensity) was performed with the global and target analysis program Glotaran, http://glotaran.org/.

After a first singular value decomposition of the raw data matrix, the starting number of components was estimated. From the first analyses with a sequential global model, the chirp corrections and the main decay/rise time constants were obtained. The number of components was further optimized. On the basis of the spectral shape (and experience), a target model was set up that contains branching from specific states. Then the ratios of this branching were introduced which influence the amplitude of the speciesassociated difference spectra (SADS), but not their shape or the decay/rise time constants. The most important spectral assumptions in the target analysis are the equality based on the singlet and triplet features of the polymer and the radicalcation spectrum of the polymer (see also Figure 2).

All spectra (measured at 256 wavelengths) were collated in a matrix, which was globally fitted using a sequential kinetic scheme with increasing lifetimes. From this, the lifetimes and the evolution-associated difference spectra (EADS) were estimated. The quality of the fit was judged by inspection of the singular vectors of the matrix of residuals, which had to be of less structure. The instrument response function was described by a Gaussian shape, and the white-light dispersion over the spectral range was modeled as a second-order polynomial. With increasing lifetimes, and thus decreasing rates, the first of the EADS decays with the first lifetime and corresponds to the difference spectrum at time zero with an ideal, infinitely small instrument response function. The second of the EADS is formed with the first lifetime and decays with the second lifetime and so on. The error in the lifetimes obtained from the fitting procedure does not exceed $10 \%$. EADS may not represent pure species, and they are interpreted as a weighted sum (with only positive contributions) of species-associated difference spectra (SADS).

To resolve the SADS from the EADS, a target analysis was performed on the data. In this target analysis, a kinetic 
scheme was used to estimate the microscopic rate constants and SADS of the different species.

The matrices of residuals resulting from the target analysis were further analyzed using a singular value decomposition. The first left and right singular vectors show no or little structure, indicating that all kinetics are described satisfactorily.

2.3.1. Quantum Chemical Calculations. We have calculated the triplet level of a DPP tetramer at $0.81 \mathrm{eV}$ with TDDFT/B3LYP with the 6-31G(d) basis set. Full geometry optimization of the $\mathrm{DPP}_{4}$ molecules were performed at the DFT level with Becke's three-parameter functional and the Lee-Yang-Parr functional (B3LYP) level associated with the 6-31G(d) basis set [20]. Time-dependent (TD) B3LYP calculations are performed for the vertical excitation energies of the lowest 5 singlet excited states, the first triplet state, and their related configuration schemes with the $6-31 \mathrm{G}(\mathrm{d})$ basis set. A developmental version of the quantum chemistry package Q-Chem was used for all calculations in the present work [21].

\section{Results and Discussion}

The steady-state UV-Vis absorption spectra of the pristine polymer and the blends have been reported previously [14] and show an absorption onset at $810 \mathrm{~nm}$. Therefore, fs-TA was performed in the NIR region on a polymer solution $\left(\mathrm{CHCl}_{3}\right)$, a pristine polymer film, a PCBM blend with a coarse morphology (code COARSE, PCE $=2 \%$, with $200 \mathrm{~nm}$ domains), and a PCBM blend with a fine morphology (code FINE; the fine morphology results in a $\sim 2 \times$ higher power conversion efficiency, $\mathrm{PCE}=4.6 \%$, with $20 \mathrm{~nm}$ domains). A laser excitation fluence of $4 \mu \mathrm{J} / \mathrm{cm}^{2}$ (at $530 \mathrm{~nm}$ ) was used. This corresponds to $1.07 \times 10^{13}$ photons $/ \mathrm{cm}^{2}$ or $1.4 \times 10^{17}$ photons $/ \mathrm{cm}^{3}$ for a $85 \mathrm{~nm}$ thick film with an OD at $530 \mathrm{~nm}$ of 0.1 . Clearly, the initial exciton density approaches charge carrier densities comparable to those in typical organic solar cells under normal operation (approximately $10^{16}$ to $10^{17} \mathrm{~cm}^{-3}$ ) [22]. In order to simulate normal operating conditions, low photon densities have to be used resulting in conditions where the signal intensity approaches a few milli-OD.

The data were fitted with a global and target analysis method [23-25] using the Glotaran software package. Since our time-window of observation is $3.6 \mathrm{~ns}$ we approximate the kinetics with exponential functions (using a rate-(K)matrix) and disregard the second-order kinetic components (see Section 2 for details). Although approaches involving distributed decay kinetics [26] or analytic solutions to rate equations [27] are available, this approximation with exponential functions is often applied for the early timescale $[28,29]$ and gives a good fit and results. We recently published a target model analysis using Glotaran of a perylene-bis(dicarboximide)/pyrene blend as well as of an annealed PCBM/P3HT film [46].

Figure 3 shows a selection of the spectra of all datasets obtained for the three materials and Tables 1 and 2 display the results of the data analysis. Triplet formation by non-geminate charge recombination of free-charge carriers that are created in $\sim 0.2 \mathrm{ps}$ implies the intermediacy of bound electron hole pairs (also called polaron pairs). In this work, we refer to these states as charge transfer state (CT states).

3.1. Pristine Polymer Films. For the pristine polymer in solution and (at low fluence) for the thin film, we observe a virtually structureless flat excited state absorption spectrum in the $900-1350 \mathrm{~nm}$ region, attributed to the singlet excited state (Figure 3(i) and Figure 4(a)). Two singlet excited state lifetimes are observed for the film (2.5 and $41 \mathrm{ps})$. These are attributed to two excitonic states with different effective conjugation lengths present in the solid film. In the solution, the longest lifetime (253 ps) is attributed to free solvated polymer molecules whereas aggregated species that are also present have a shorter (22 ps) lifetime. Previous studies have indicated that this polymer partly aggregates in solution [14]. The $1 \mathrm{ps}$ component is attributed to solvation processes. A very minor amount of triplet state formation $(\sim 5 \%)$ is observed for the thin film. The triplet excited state spectrum of the DPPP polymer was previously reported, observed with steady-state PIA, [14] and shows a maximum at $\sim 1100 \mathrm{~nm}$ (see also Figure 2). Singlet-singlet, triplet-triplet, CT-CT, and CT-exciton annihilation are known to play a role in fs-TA spectroscopy of thin-film blends $[30,31]$. In the simplest approximation, annihilation processes are known to display a quadratic dependence on the laser fluence (LF) [32]. Such a fit (Figure 4(e)) gives a relative efficiency of triplet formation $\left(\eta_{\mathrm{T}}\right)$ value of $3 \%$ :

$$
\lim _{\mathrm{LF} \rightarrow 0} \eta_{\mathrm{T}}=0.03 \text {. }
$$

The various lifetimes and maximum populations of triplet formation are reported in Table 1. The laser fluence effects on these quantities are visualized in Figure 4(e). The lifetimes of the singlet excited states become longer upon lowering the laser fluence (from 1 to $41 \mathrm{ps}$ ). The relative triplet yield shows a similar trend, less triplet is formed at lower laser fluence (from 0.43 to 0.05 ). The triplet excited state lifetime shows dependence on the fluence, going from $1.1 \mathrm{~ns}$ (at $28 \mu \mathrm{J} / \mathrm{cm}^{2}$ ) to more than $3.6 \mathrm{~ns}$ (at $4 \mu \mathrm{J} / \mathrm{cm}^{2}$ ) at low fluence. It has to be noted that our observation window is $3.6 \mathrm{~ns}$, and therefore, this latter value is on the limit of our detection. Clearly, the triplet state has a much longer lifetime, as it was observed previously with steady-state PIA for a similar film [14]. The $1.1 \mathrm{~ns}$ lifetime at a higher laser fluence is influenced by triplet-triplet annihilation. The singlet excited state lifetime shows a sharper dependence and so does the relative triplet yield. The complex photophysical behavior with respect to the laser fluence of the pristine polymer films is reflected in the properties of the blends.

3.2. Thin-Film Blends. For the blend films at low laser fluence, we observe a short-lived singlet-excitonic state that converts rapidly to a charged state in $\sim 0.2 \mathrm{ps}$, characterized by the positive polaron absorption. The electrochemically oxidized PDPP has been reported earlier and shows a typical absorption band at $1350 \mathrm{~nm}$ (and a shoulder at $900 \mathrm{~nm}$, sloping 

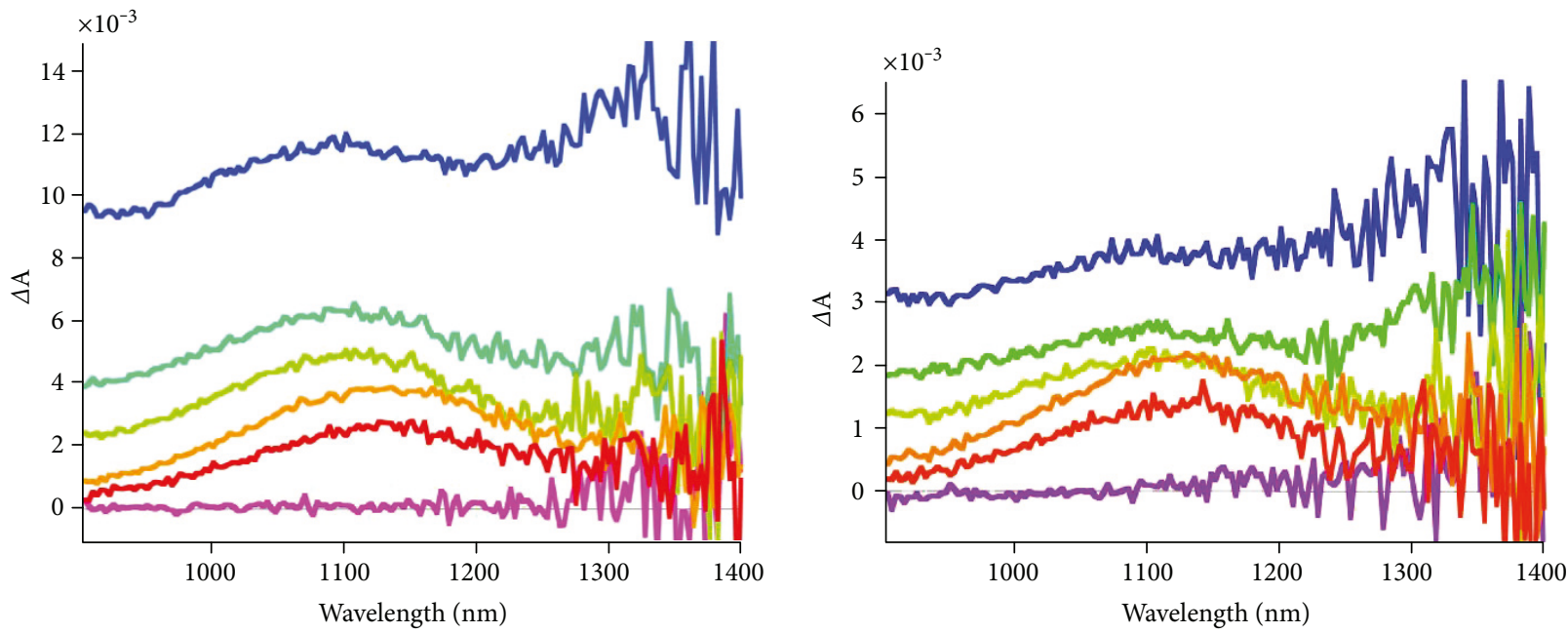

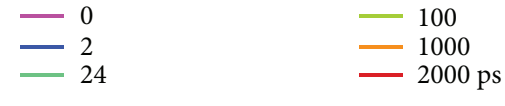

(a) FINE blend, $28 \mu \mathrm{J} / \mathrm{cm}^{2}$

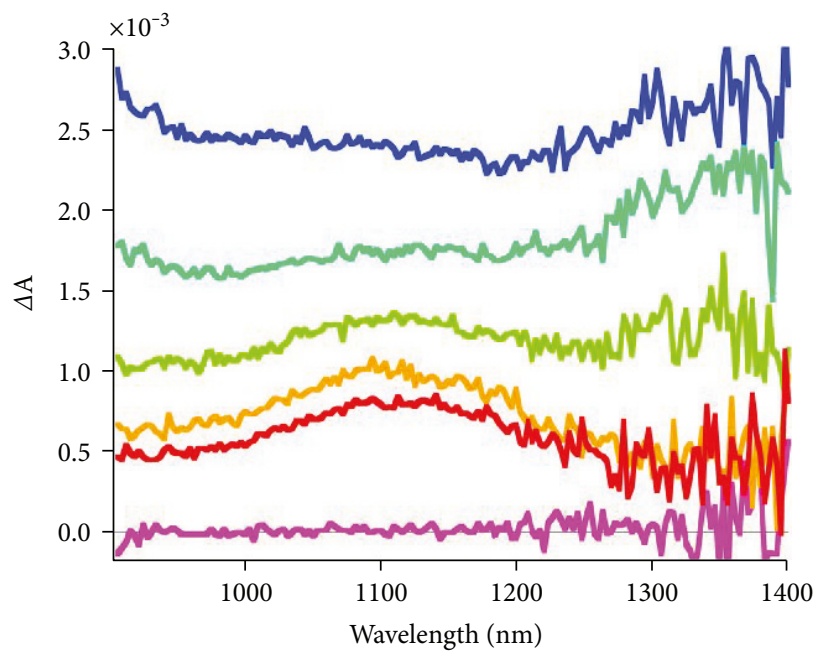

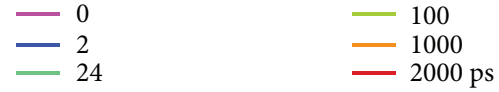

(b) FINE blend, $13 \mu \mathrm{J} / \mathrm{cm}^{2}$
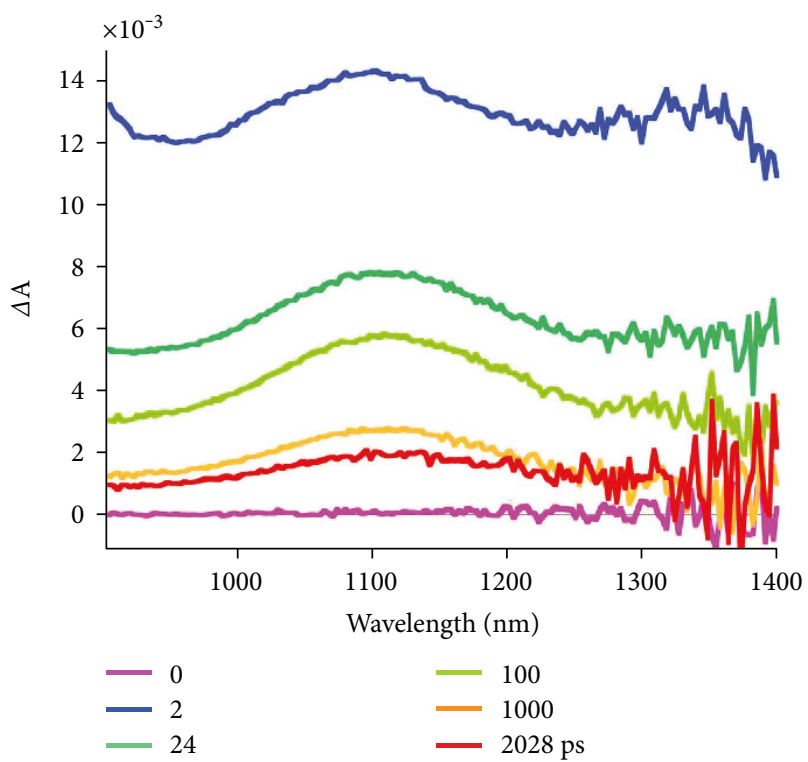

(d) COARSE blend, $28 \mu \mathrm{J} / \mathrm{cm}^{2}$

(c) FINE blend, $4 \mu \mathrm{J} / \mathrm{cm}^{2}$

Figure 3: Continued. 

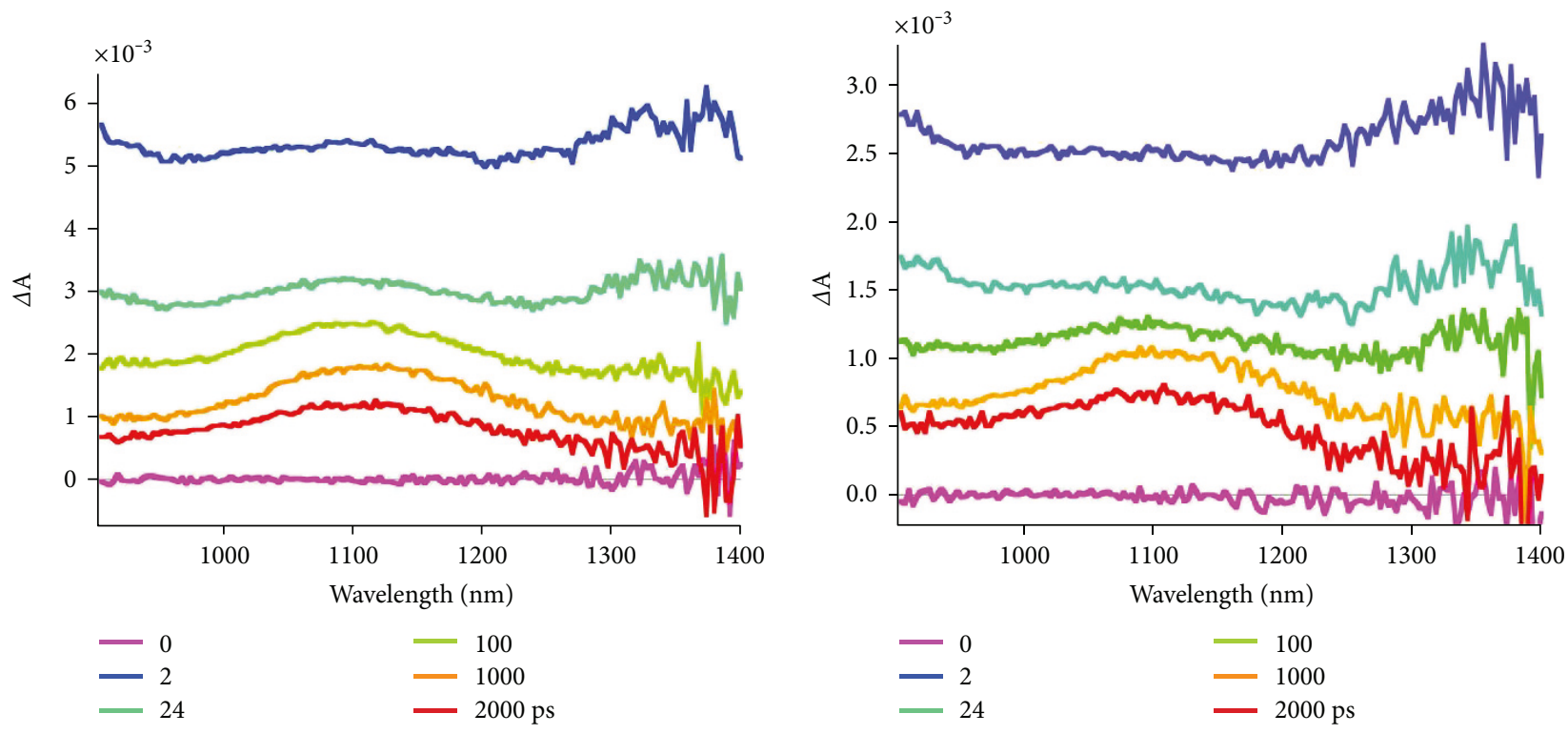

(e) COARSE blend, $13 \mu \mathrm{J} / \mathrm{cm}^{2}$
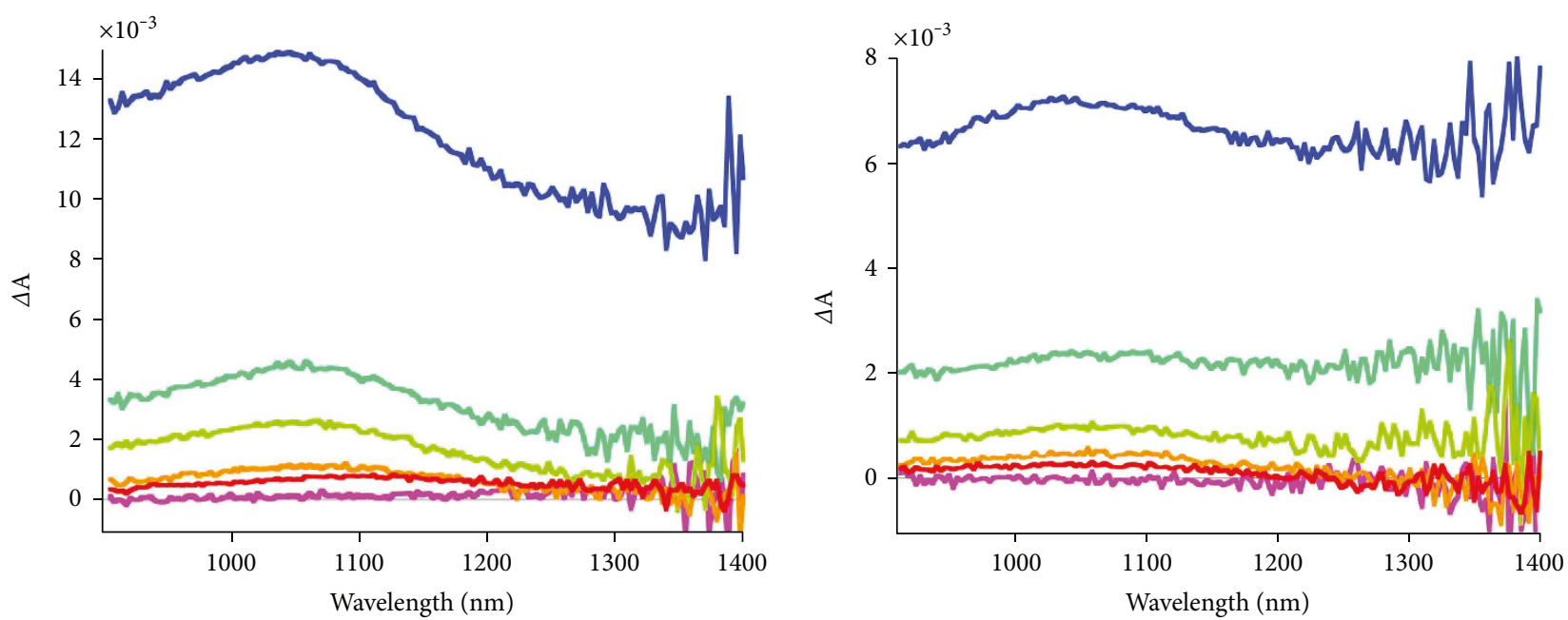

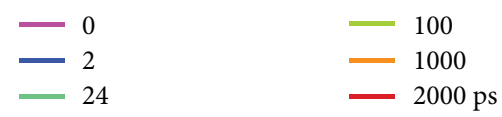

(g) Polymer, $28 \mu \mathrm{J} / \mathrm{cm}^{2}$

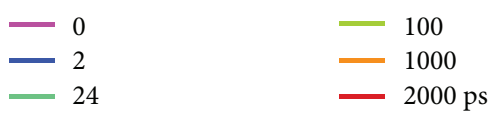

(h) Polymer, $13 \mu \mathrm{J} / \mathrm{cm}^{2}$

Figure 3: Continued. 


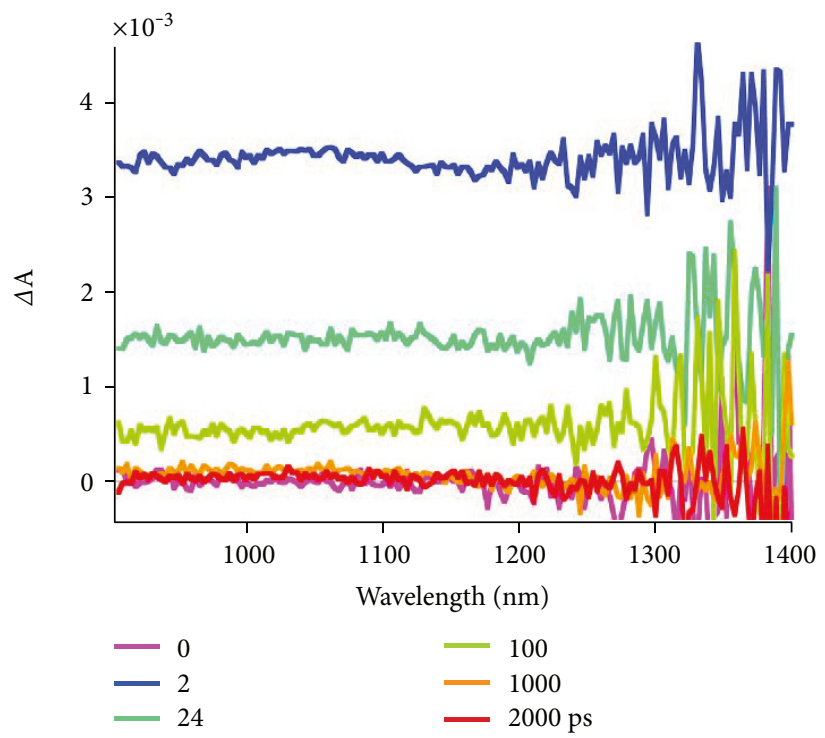

(i) Polymer, $4 \mu \mathrm{J} / \mathrm{cm}^{2}$

Figure 3: Displayed are selected fs-TA spectra of thin films at various delay times and at different laser powers: (a, b, c) FINE blends (using cosolvents ODCB/DIO); (d, e, f) COARSE blends obtained from $\mathrm{CHCl}_{3} ;(\mathrm{g}, \mathrm{h}, \mathrm{i})$ pristine PDPP polymer films. Delay times are indicated. Excitation was performed at $530 \mathrm{~nm}$. Note: if we go from (a) to (b) to (c) (or from (d) to (e) to (f) or from (g) to (h) to (i)) the relative amount of ultrafast triplet formation ( $1100 \mathrm{~nm}$ band) by singlet fission is reduced at early times; if we go from (g) to (d) to (a) (or from (h) to (e) to (b) or form (i) to (f) to (c)) the relative amount of ultrafast charge generation is increased (1350 nm band).

down from the blue side of the spectral range, see also Figure 2), clearly shifted relative to the triplet state absorption at $1100 \mathrm{~nm}$. Analysis of the data indicates that now, the main part of the charged species recombines to form the triplet excited state of the polymer. As can readily be seen in the spectra shown in Figure 3, the band at $1100 \mathrm{~nm}$ develops as the $1350 \mathrm{~nm}$ band decays.

The six data sets of the blends (the FINE and COARSE morphology at three light intensities) were analyzed with the global and target analysis method (Glotaran).

The main outcome of the target analysis for the PDPP/ PCBM blends is presented in Figure 5 and Table 2. Next to the most important time constants, we obtain the so-called species-associated difference spectra (SADS). These are the spectra belonging to specific species that play a role in the light-induced processes. In order to describe the matrix of time resolved spectra appropriately, we need two species with a "singlet" character, two species with charges, and one triplet state. A characteristic target model is shown in Figure 5(a), in which, for example, the transition from $\mathrm{A}$ to $\mathrm{E}$ is the singlet fission. The relative contribution of this particular channel will be influenced by laser power and morphology. This is the case for all branching pathways within the target model in Figure 5(a). Figure 5(b) shows the populations versus time of the various excited state species of which the spectra are displayed in Figure 5(c) (species-associated difference spectra, SADS). Within the analysis, the shape of the SADS is a main focus. Separating triplet contributions (1100 $\mathrm{nm}$ band) from charge transfer state contributions (1350 $\mathrm{nm}$ band) is an important argument when moving from a simple sequential global analysis (e.g., $\mathrm{A}>\mathrm{B}>\mathrm{C}$ ) to a target model (with branching).

Figure 5 and Table 2 show how the photophysics is influenced by morphology and fluence. The increase in efficiency of charge formation with fluence reduction is stronger for the FINE film, accompanied by a reduction in relative triplet formation. The lifetime of the charges increases more for the FINE film, upon fluence reduction, as compared to the COARSE film. The effects of the laser fluence effects and the morphology can be understood by a kinetic competition of hot singlet fission and CT formation.

If we assume a quadratic dependence on the laser fluence, a fit results in $72 \%$ CT and 38\% triplet for the FINE morphology, as values extrapolated to zero fluence.

For the COARSE morphology, these values are $63 \%$ CT and $48 \%$ triplet. Since a negligible amount of triplet is formed in the pristine polymer at low fluence, the ratios of the above values quantify the anticipated charge loss at solar conditions $(38 / 72) \times 100=52 \%$ charge loss for the FINE morphology and $76 \%$ charge loss for the COARSE morphology. This would imply loss-corrected PCE values of 9.6 and $8.3 \%$.

Clearly, the $\eta_{\mathrm{T}}$ and $\eta_{\mathrm{CT}}$ are strongly influenced by annihilation processes (resulting in triplet formation) that compete with free-charge generation.

A linear fit to the lifetimes gives extrapolated values of 968 and $323 \mathrm{ps}$ for $\tau(\mathrm{CT})$ FINE and COARSE. Triplet lifetimes of 6.1 and $3.7 \mathrm{~ns}$ were obtained for the FINE and COARSE blends. The linear fit (see Figure 5(e)) indicates that 
TABLE 1: Effects of laser fluence (in $\mu \mathrm{J} / \mathrm{cm}^{2}$ ) on the maximum populations of triplet state formation $\left(\eta_{\mathrm{T}}\right.$ ) together with the lifetimes of triplet states $\left(\tau_{\mathrm{T}}^{n}\right)$ and singlet states $\left(\tau_{\mathrm{S} 1}^{n}\right)$ obtained for pristine polymer films as well as values obtained for the polymer in $\mathrm{CHCl}_{3}$ solution.

\begin{tabular}{|c|c|c|c|c|c|c|}
\hline Laser fluence $\left(\mu \mathrm{J} / \mathrm{cm}^{2}\right)$ & $\eta_{\mathrm{T}}$ & $\tau_{T}^{1}(\mathrm{~ns})$ & $\tau_{\mathrm{T}}^{2}(\mathrm{ps})$ & $\tau_{\mathrm{S} 1}^{1}(\mathrm{ps})$ & $\tau_{S 1}^{2}(\mathrm{ps})$ & $\tau_{\mathrm{S} 1}^{3}(\mathrm{ps})$ \\
\hline 28 & 0.43 & 1.2 & 20 & 1.0 & - & \\
\hline 12 & 0.10 & 3.3 & - & 2.1 & 29 & \\
\hline 4 & 0.05 & 3.6 & - & 2.5 & 41 & \\
\hline \multicolumn{7}{|l|}{$\left(\mathrm{In} \mathrm{CHCl}_{3}\right)$} \\
\hline 28 & & & & 1 & 22 & 253 \\
\hline
\end{tabular}

TABLE 2: Effects of laser fluence (in $\mu \mathrm{J} / \mathrm{cm}^{2}$ ) on maximum populations of triplet state formation $\left(\eta_{\mathrm{CT}}\right.$ ) and total charge transfer state formation $\left(\eta_{\mathrm{T}}\right)$ together with the lifetimes of CT states $\left(\tau_{\mathrm{CT}}\right)$ and triplet states $\left(\tau_{\mathrm{T}}\right)$ as well as the rise times for the triplet states $\left(\tau^{n}{ }_{\mathrm{T}}{ }^{\text {rise }}\right)$ obtained for COARSE as well as for FINE blend films. Also shown are the lifetimes of the Franck-Condon state $\left(\tau_{\mathrm{FC}}\right)$, of the hot singlet state $\left(\tau^{*}{ }_{\mathrm{S} 1}\right)$, and of the hot CT state $\left(\tau^{*}{ }_{\mathrm{CT}}\right)$.

\begin{tabular}{|c|c|c|c|c|c|c|c|c|c|c|}
\hline Laser fluence $\left(\mu \mathrm{J} / \mathrm{cm}^{2}\right)$ & $\eta_{\mathrm{CT}}$ & $\eta_{\mathrm{T}}$ & $\tau_{\mathrm{FC}}(\mathrm{ps})$ & $\tau_{\mathrm{S} 1}^{*}(\mathrm{ps})$ & $\tau^{*}{ }_{\mathrm{CT}}(\mathrm{ps})$ & $\tau_{\mathrm{CT}}(\mathrm{ps})$ & $\tau_{\mathrm{T}}(\mathrm{ns})$ & $\tau_{\mathrm{T}}^{1}{ }^{\text {rise }}(\mathrm{ps})$ & $\tau_{\mathrm{T}}^{2}{ }^{\text {rise }}(\mathrm{ps})$ & $\tau_{\mathrm{T}}^{3}$ rise $(\mathrm{ps})$ \\
\hline \multicolumn{11}{|l|}{ COARSE } \\
\hline 28 & 0.20 & 0.80 & 0.22 & 0.52 & 4.26 & 52 & 2.0 & 0.52 & 4.26 & 52 \\
\hline 12 & 0.55 & 0.55 & 0.29 & 2.56 & 22 & 126 & 2.9 & & 22 & 126 \\
\hline 4 & 0.60 & 0.5 & 0.28 & 2.4 & 37 & 318 & 3.4 & & 37 & 318 \\
\hline \multicolumn{11}{|l|}{ FINE } \\
\hline 28 & 0.20 & 0.80 & 0.20 & 0.39 & 5.5 & 60 & 2.5 & 0.39 & 5.5 & 60 \\
\hline 12 & 0.62 & 0.45 & 0.29 & 2.86 & 40 & 410 & 4.5 & & 40 & 410 \\
\hline 4 & 0.7 & 0.4 & 0.20 & 1.25 & & 900 & 5.5 & & & 900 \\
\hline
\end{tabular}

these decays are not governed by annihilation but by, for example, a constant concentration of trap states present within the thin-film blend.

The femtosecond spectroscopy presented here can be correlated to the spectra of the triplet state obtained by ns spectroscopy and spectro-electrochemistry that have been reported before. Figure 2 reports these spectra (from reference [14]) that have been converted to a wavelength scale for clarity.

3.3. Mechanistic Aspects. Non-geminate bimolecular recombination coefficients $(\beta)$ in polymer/fullerene solar cells have been determined to be $6 \times 10^{-11} \mathrm{~cm}^{3} \mathrm{~s}^{-1}$ (for PDPP-5T/ PCBM) [33], $3 \times 10^{-12} \mathrm{~cm}^{3} \mathrm{~s}^{-1}$ and $2 \times 10^{-13} \mathrm{~cm}^{3} \mathrm{~s}^{-1}$ (both for $\mathrm{P} 3 \mathrm{HT} / \mathrm{PCBM})[34,35]$. This correlates to decay times between $60 \mathrm{~ns}$ and $1.6 \mu \mathrm{s}$ at the lowest laser fluence used in this study (assuming 100\% initial charge generation). This is slower than the recombination rates observed here.

Charge recombination to the triplet state has been studied in many molecular electron donor acceptor systems, [36-41] and these studies can be correlated to our results. To estimate the energetics of our PDPP/PCBM system, we can use the data $E_{\mathrm{ox}}=+0.25 \mathrm{~V}$ versus $\mathrm{Fc} / \mathrm{Fc}^{+}$in $\mathrm{ODCB}$, $E_{\text {red }}=-1.07 \mathrm{~V}$ versus $\mathrm{Fc} / \mathrm{Fc}^{+}$in $\mathrm{ODCB}$, and $E_{\mathrm{S} 1}=1.53 \mathrm{eV}$. For the triplet state level, we can use values from a study of molecular model systems [42] giving a triplet level of DPP-T2 at $0.9 \mathrm{eV}$. We have calculated a triplet level of a DPP tetramer at $0.81 \mathrm{eV}$ with TDDFT/B3LYP with the $6-31 \mathrm{G}(\mathrm{d})$ basis set. We thus take $E_{\mathrm{T}}=0.85 \mathrm{eV}$ as the average estimated value. The dielectric constant present in the solid can be taken to be 4 [43-45].
We can also estimate the energy of the CT density of states by using the open circuit voltage and the " $E_{\mathrm{g}}$ rule" to give $e\left(V_{\mathrm{OC}}+E_{\mathrm{g}}\right)$ resulting in a value between 1.18 and $1.28 \mathrm{eV}$. This is just above the anticipated triplet energy level and correlates well with the values obtained for molecule model systems (DPP-T2/C60 Ecss $=1.52$ to $1.32 \mathrm{eV}$ ) [42].

Within the Marcus theory of electron transfer, spin is not taken into account. Clearly, a recombination to the local triplet state must be accompanied by an electron spin flip. The compensating magnetic momentum change in intersystem crossing (ISC) can operate through spin orbital coupling (SOC) or through proton hyperfine interactions (H-HFI).

For molecular diketopyrrolopyrrole-oligothiophene(T)fullerene triads [42] (DPP-T2- $\mathrm{C}_{60}$ in toluene), we estimated the intrinsic intersystem crossing time constant to be $\sim 15 \mathrm{~ns}\left(k_{\mathrm{ISC}}=6.7 \times 10^{7} \mathrm{~s}^{-1}\right)$. This was reported to be the rate-limiting step for (triplet) charge recombination in this molecular system studied in solution. For the shorter molecular DPP-T1-C 60 (with a larger exchange integral, $J$, see later) $0.8 \mathrm{~ns}$ in ortho-dichlorobenzene was found [42]. Recently, we showed fast and slow rates for charge recombination [46] to the triplet state in perylene bisimide/pyrene thin-film blends $\left({ }^{3} k_{\mathrm{CR}}=1.04 \times 10^{10}\right.$ and $\left.{ }^{3} k_{\mathrm{CR}}=7.21 \times 10^{7} \mathrm{~s}^{-1}\right)$.

For molecular model systems in solution, two mechanisms can be responsible for ISC in charge transfer states: spin-orbit charge-transfer ISC (SOCT-ISC) and radical-pair ISC (RP-ISC) [52-57]. In SOCT-ISC, the change of electron magnetic momentum needed for the electron flip is compensated by the change of orbital magnetic momentum that occurs when an electron is moved from one orbital to 


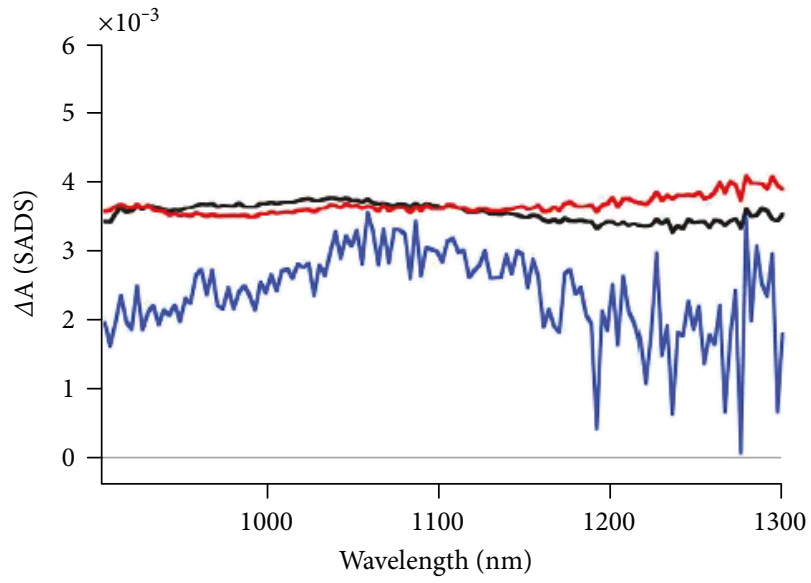

(a) Polymer SADS, $4 \mu \mathrm{J} / \mathrm{cm}^{2}$

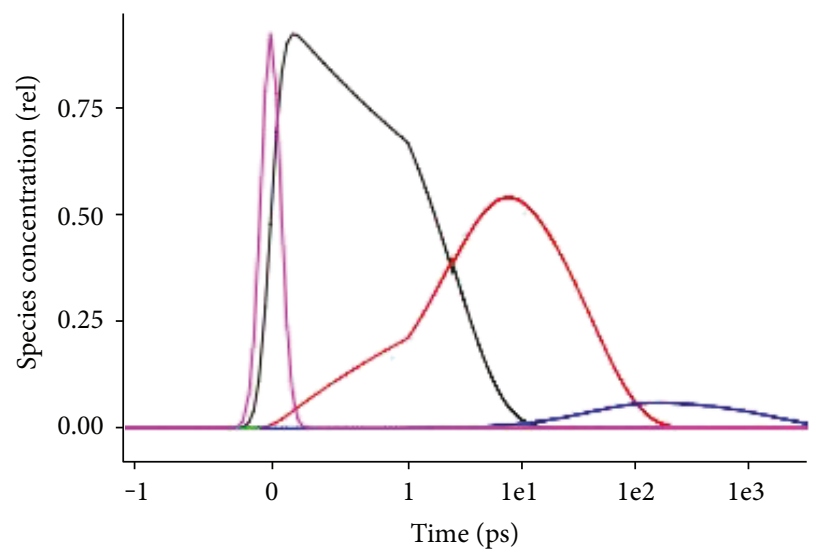

(c) Polymer kinetics, $4 \mu \mathrm{J} / \mathrm{cm}^{2}$

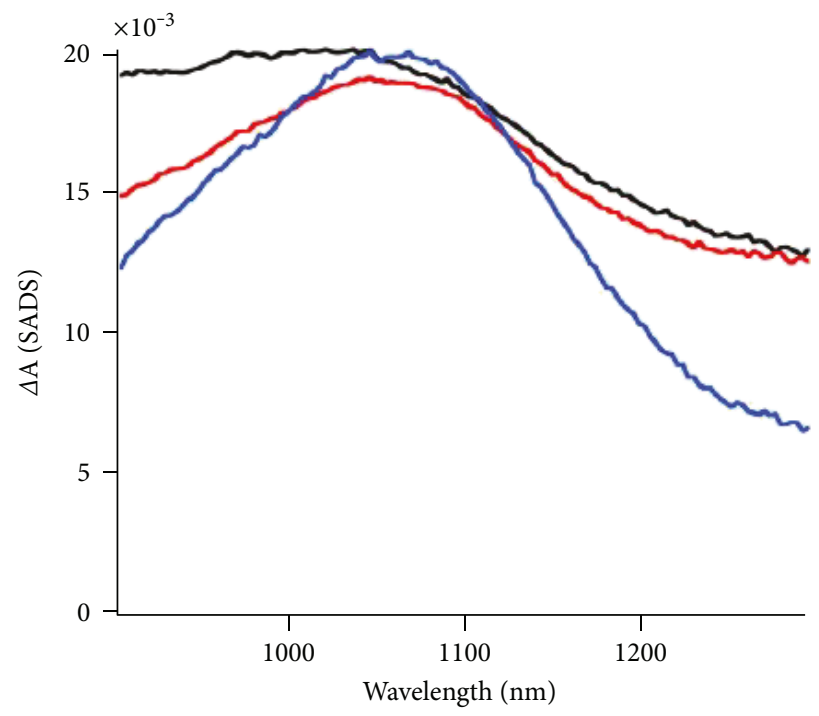

(b) Polymer SADS, $28 \mu \mathrm{J} / \mathrm{cm}^{2}$



(d) Polymer kinetics, $28 \mu \mathrm{J} / \mathrm{cm}^{2}$

Figure 4: Continued. 




(e) Effect of fluence

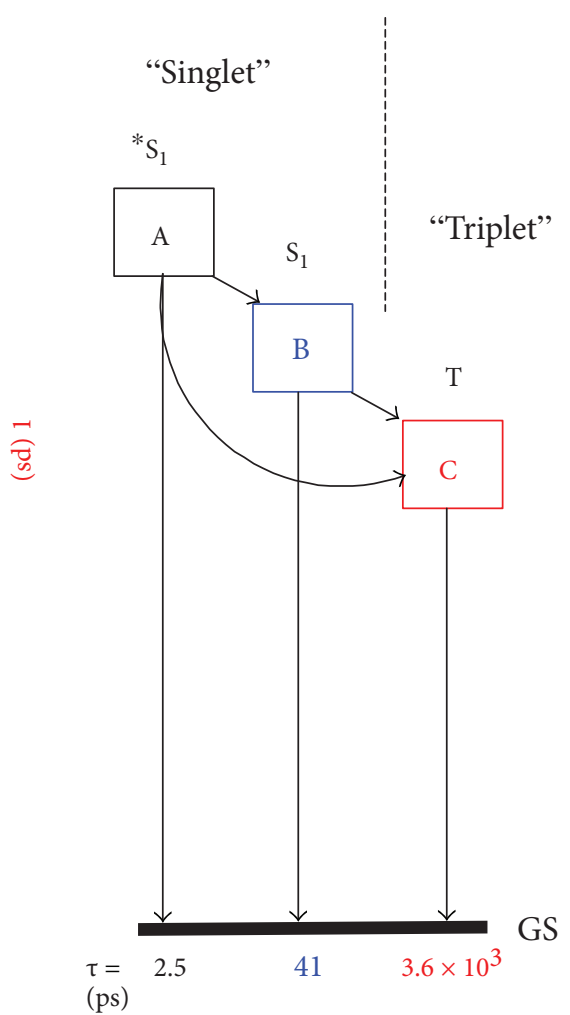

(f) Target model

FIgURe 4: fs-TA data analysis of the pristine polymer films at low laser fluences $(a, c)$ and at high laser fluences (b, d). (a, b) Species-associated difference spectra obtained from the target analysis (for (a), black is hot singlet, red is relaxed singlet, and blue is triplet; for (b), black is hot singlet, red is hot triplet, and blue is relaxed triplet). (c, d) Concentration profiles of the species reported in ( $a, b)$. The pulse is given in pink (in (c)) or in green (in (d)). (Note the lin-log timescale axis in (c) and (d).) (e) Laser fluence effects on the photophysical properties of the pristine polymer film. Shown is the change of singlet and triplet excited state lifetimes and of the relative triplet formation efficiency as function of laser fluence for the pristine polymer films. (f) Target model used for the analysis for low fluence (for high fluence, the three species were hot singlet, hot triplet, and relaxed triplet).

another. If the symmetry of these orbitals is different or if their main orbital coefficients lie in different regions in space (if they are for instance orthogonal), this is beneficial for SOCT-ISC. Studies by Colvin et al. have given clear evidence for this mechanism [52-57]. RP-ISC proceeds via proton hyperfine interactions (H-HFI). The nuclear spin momentum of the hydrogen atoms have to adjust in such a way that magnetic momentum compensation occurs, within the radial pair, converting a singlet CT state into a triplet CT state. This state can then recombine to the local triplet state without spin forbiddenness. So far, we cannot discriminate between these two modes of operation but using a deuterated polymer should be an interesting approach. We suggest a model in which optimized morphology is correlated to a CT state with a smaller exchange integral and a reduced contribution of SOCT-ISC (see Figure 7). This model is in line with recent results obtained by using the photo-induced Stark shift [47], indicating a larger distance of charge separation in optimized blends as studied by Gélinas et al. The correlation between electron and hole distance, the energy difference between singlet and triplet, the relative contribution of spin orbit coupling, and the contribution of the radical pair mechanism was discussed before [48].

The ultrafast (ps) triplet generation we observe here and the strong influence of the laser power on this process indicate that exciton annihilation is responsible [19, 49].

Spin conservation rules dictate that it is spin forbidden to generate one triplet (and a ground state singlet). Thus, hot singlet fission is the only viable option for such ultra-fast triplet state formation. Furthermore it is important to note that, in general, singlet fission occurs in very well ordered crystalline phases. Interestingly, there have also been reports on singlet fission in spin-coated regio-random poly-thiophene films [50].

\section{Discussion and Conclusions}

At low laser fluence (approaching solar intensity), charge separation dominates in PDPP/PCBM blends. Triplet formation by charge recombination (Figure 6) is an important decay channel of the charge transfer state and morphology influences this process. 


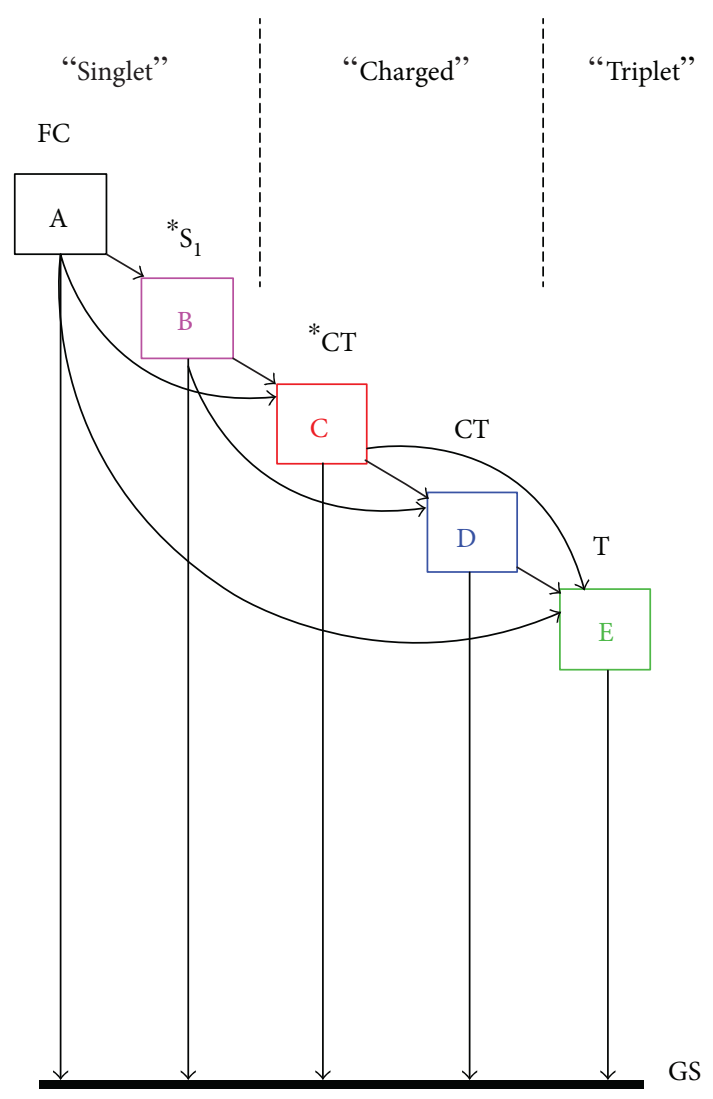

(a) Target model

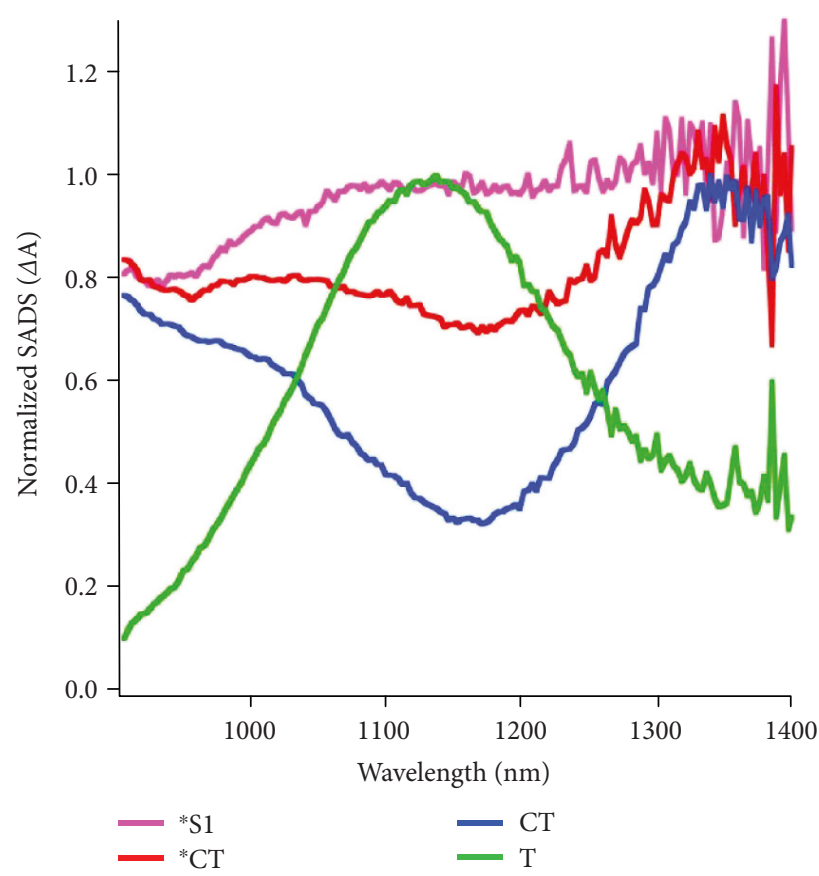

(c) SADS

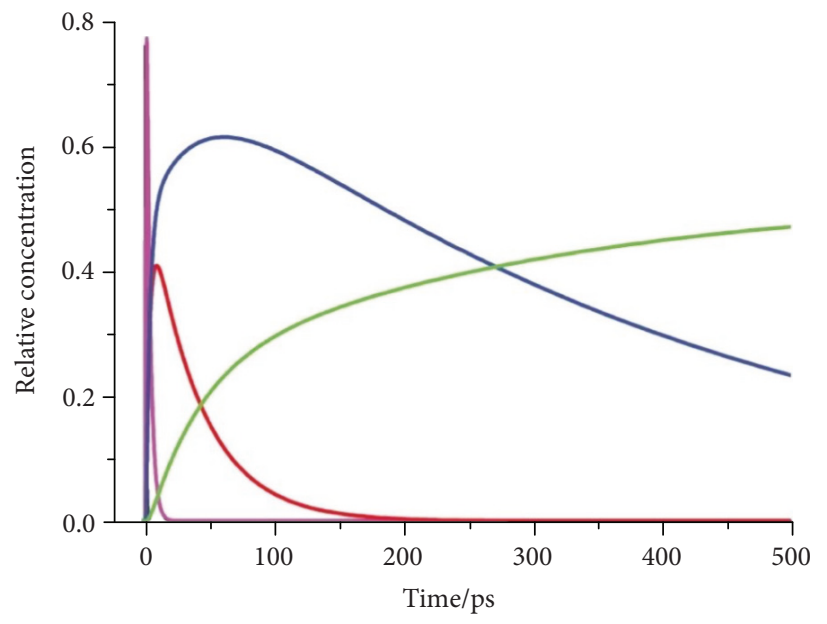

(b) Kinetics of SADS



(d) Fluence effects on yields

Figure 5: Continued. 


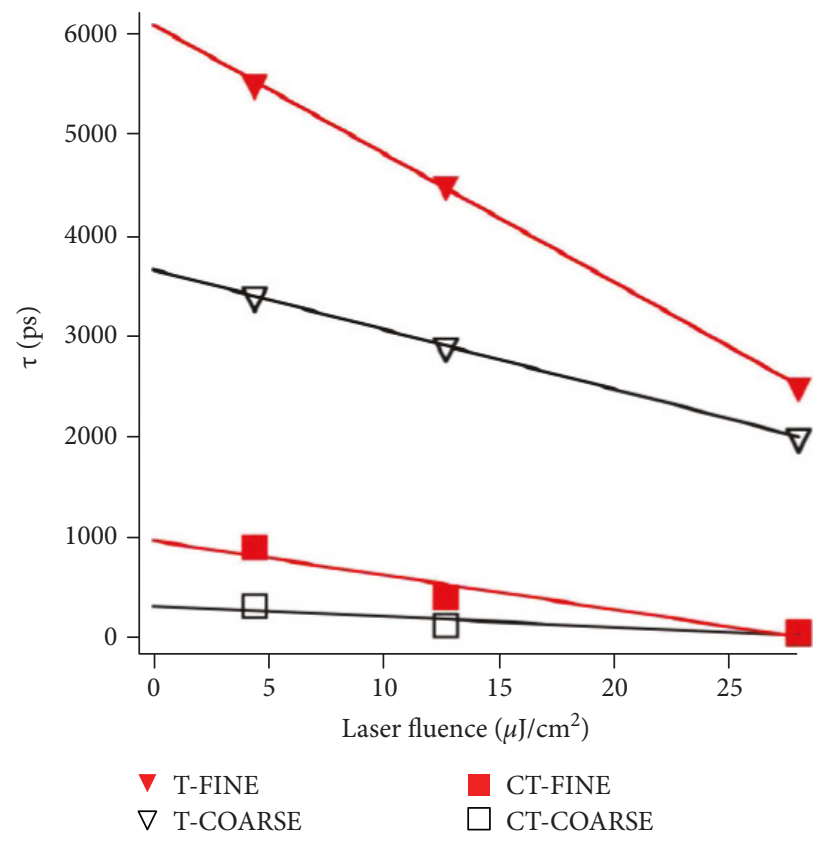

(e) Fluence effects on decay times

FIGURE 5: fs-TA data analysis of the blend films. (a) Representative target model used for the analysis. Note the multiple branching pathways. The transition from A to E is singlet fission. See below for key. (b) Representative concentration profiles of the species-associated difference spectra (SADS) obtained for the FINE sample (cosolvent is ODCB) using a laser fluence of $12 \mu \mathrm{J} / \mathrm{cm}^{2}$. Decay times (in ps) belonging to the various species are $0.29(\mathrm{FC}), 2.86\left({ }^{*} \mathrm{~S} 1\right), 40\left({ }^{*} \mathrm{CT}\right), 410(\mathrm{CT})$, and $4.5 \mathrm{~ns}(\mathrm{~T})$; see below for key. (c) Representative normalized SADS obtained for the FINE sample (cosolvent is ODCB) using a laser power of $28 \mu \mathrm{J} / \mathrm{cm}^{2}$. Decay times (in ps) belonging to the various species are $0.39\left({ }^{*} \mathrm{~S} 1\right), 5.5\left({ }^{*} \mathrm{CT}\right), 60(\mathrm{CT})$, and $2.5 \mathrm{~ns}$ (T). FC species is not shown. (d) Relative triplet and charge transfer state formation efficiency as functions of laser fluence for the COARSE and the FINE blend films, with 2nd-order polynomial fit. (e) Change of triplet and charge transfer excited state lifetimes as function of laser fluence for the COARSE and the FINE blend films, with linear fit. Key: Franck-Condon (FC) state $=$ black, hot $S_{1}\left({ }^{*} S_{1}\right)=$ pink, hot charge transfer state $\left({ }^{*} \mathrm{CT}\right)=$ red, charge transfer state $(\mathrm{CT})=$ blue, triplet $(\mathrm{T})=$ green. It has to be noted that the hot CT state is not a vibrational hot CT state but a collection of higher lying CT states within the CT density of states.

We show complex photophysics and fast kinetics of the spin flip dynamics to a triplet excited state occurring from the CT state in a low band gap polymer/PCBM blend at low laser fluence. The charge recombination effect to the polymer triplet state occurs on a $\sim 300$ to $\sim 900$ ps timescale, depending on film morphology, and plays a role in both PDPP/PCBM blend films. Triplet formation by charge recombination is slower in the FINE blend film of PDPP/ PCBM (with $20 \mathrm{~nm}$ domains) relative to the COARSE blend film (with $200 \mathrm{~nm}$ domains). At the low laser fluences used here, charge generation dominates and triplet formation by charge recombination occurs. If we are able to control the triplet formation by charge recombination, we can anticipate a power conversion efficiency of $9.6 \%$ for these types of materials (based on value corrected for the charge loss to the triplet state).

In polymer films (without PCBM), we see that the singlet excited state of the PDPP polymer films shows lifetimes of 2.5 and 41 ps and a virtually flat $S_{1}-S_{n}$ absorption band in the $900-1400 \mathrm{~nm}$ region (at low laser fluence).

It has been estimated that charge recombination to the triplet states with a mode of operation of proton hyperfine interactions occurs with a rate between $1 \times 10^{6}$ and $1.4 \times 10^{8} \mathrm{~s}^{-1}$ [51]. However, in molecular D-A systems studied in solution, various examples are available where ns or even sub-ns charge recombination to the triplet occurs. Spin orbit coupling coupled to charge transfer (SOCT) must be an additional important and faster factor for ISC, (e.g., $1 \times 10^{9} \mathrm{~s}^{-1}$ to $2.5 \times 10^{10} \mathrm{~s}^{-1}$ ) [41], especially if the orbitals corresponding to the transition are orthogonal [52-57]. Okada et al. observed indications for SOCT-ISC already in 1981 [41], and the basis for these effects correlates to the El-Sayed rules [54]. These types of effects are likely to play a role in the blends studied here.

We present a model that correlates nanoscale morphology with triplet formation by charge recombination based on known mechanisms for intersystem crossing. Fast SOCT-ISC dominates in strongly (coulomb) bound CT states with a large $J$ (and electronic coupling $V$ ). Slower H-HFI-ISC becomes important in charged states where $V$ and the exchange integral $(J)$ are small; see Figure 6.

Visualization of the processes occurring in the blends is shown in Figures 6 and 7.

Clearly, we need further studies on ultra-fast charge and triplet generation in low band gap polymer-PCBM blends probing the effects of morphology on triplet charge recombination (also in working devices). To attain a full understanding of the primary events in organic 


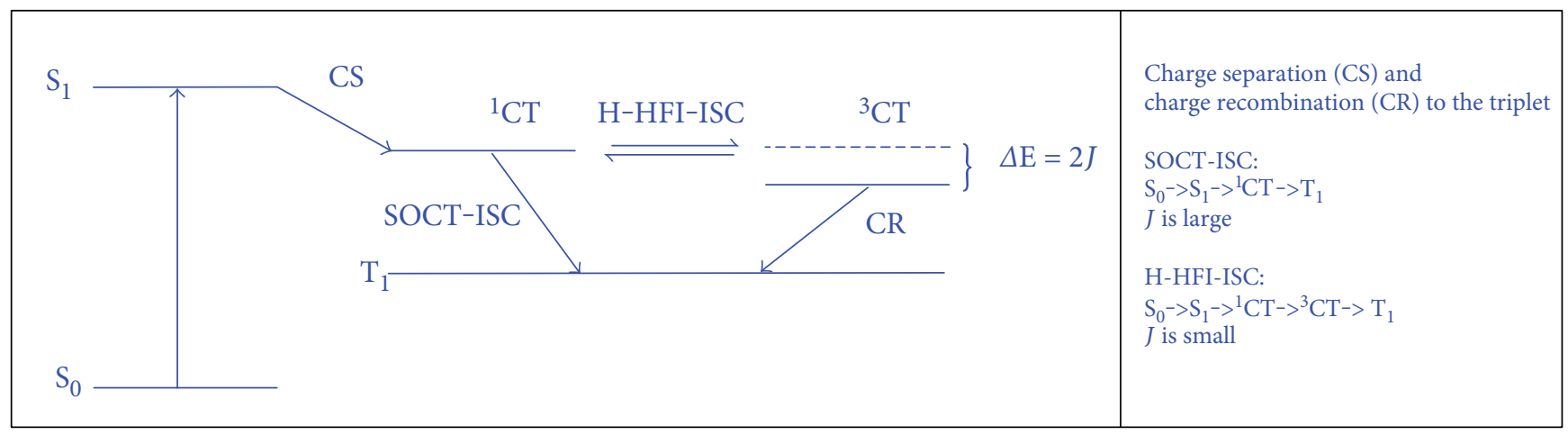

FiguRE 6: Representation of the processes described earlier for molecular donor-acceptor systems [51]: charge separation leads to a charge transfer state; geminate charge recombination to the triplet state can occur through proton hyperfine interactions (H-HFI-ISC) and through spin-orbit charge transfer intersystem crossing (SOCT-ISC). The energy difference of the singlet and triplet charge transfer state is twice the exchange integral $(J)$. See Figure 7 for application of these aspects to non-geminate charge recombination in polymer blends.

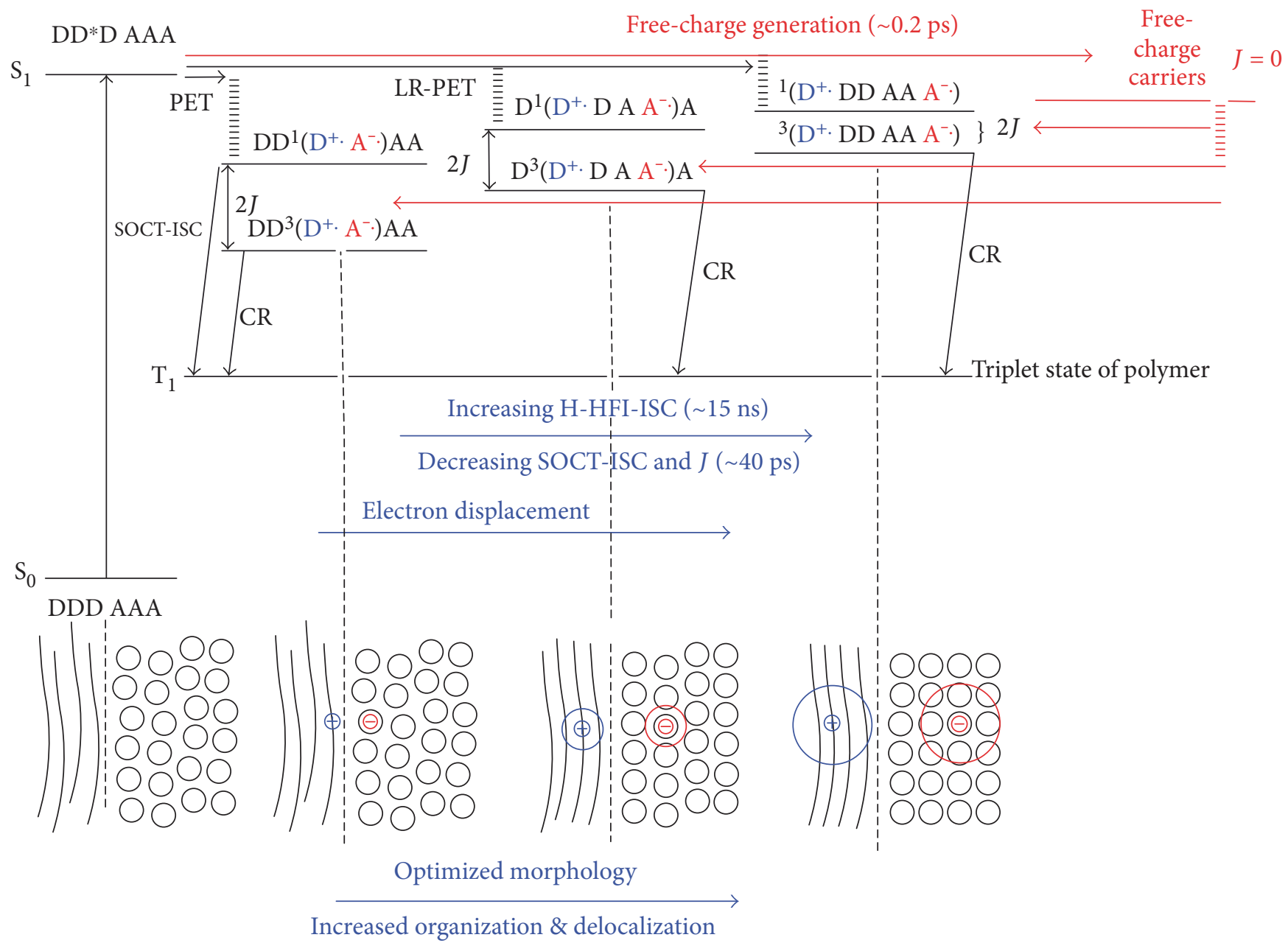

FIGURE 7: Representation of the effect of morphology on charge recombination to the triplet state. Free-charge generation (FCG) occurs in 0.2 ps. In a disordered morphology (COARSE), the charge transfer state is characterized by a larger $J$ (exchange integral) and SOCT-ISC can play a more important role. In the well-ordered almost crystalline morphology, the charge transfer state has a smaller $J$. The contribution of the slower H-HFI interactions will become more important as $J$ diminishes in size. The contribution of the faster SOCT-ISC becomes smaller upon going to the right side of the figure. For free-charge carriers (FCC), $J$ equals zero. Charge recombination from ${ }^{3} \mathrm{CT}$ to $\mathrm{T}_{1}$ is spin allowed. Note that the effect of optimized morphology (increased organization) on polaron energetics is neglected. More organization can lead to lowering of the energies of the positive polaron, the negative polaron, and the free-charge carriers (FCC) (relative to the coulomb bound charge transfer state) because of delocalization and entropy effects. In principle, photo-induced electron transfer (PET) can also populate the CT states over long ranges (LR) for the optimized morphology. 
photovoltaics, it is crucial to study and learn to control charge recombination. The use of deuterated polymers and deuterated PCBM (or pristine $\mathrm{C}_{60}$ ) should modify H-HFI-ISC. This could result in a spin tool to experimentally discriminate the ISC mechanisms occurring in OPV materials. Furthermore, studies of magnetic field effects on these types of materials will be of great importance.

\section{Conflicts of Interest}

The authors declare no competing financial interests.

\section{Authors' Contributions}

René A. J. Janssen, Stephan C. J. Meskers, and René M. Williams designed the experiments. Daniele Di Nuzzo prepared the thin films. Hung-Cheng Chen (supervised by René M. Williams) performed the time resolved measurements and analyzed the data. Hung-Cheng Chen performed the DFT calculations. René M. Williams and Hung-Cheng Chen wrote the paper.

\section{Acknowledgments}

The authors are grateful to NWO (de Nederlandse Organisatie voor Wetenschappelijk Onderzoek) for the grant for the femtosecond equipment and the UvA (Universiteit van Amsterdam) for the structural support. They thank Caspar Williams and Lisette van Hooijdonk for language corrections.

\section{References}

[1] N. S. Sariciftci, L. Smilowitz, A. J. Heeger, and F. Wudl, "Photoinduced electron transfer from a conducting polymer to buckminsterfullerene," Science, vol. 258, no. 5087, pp. 14741476, 1992.

[2] G. Yu, J. Gao, J. C. Hummelen, F. Wudl, and A. J. Heeger, "Polymer photovoltaic cells: enhanced efficiencies via a network of internal donor-acceptor heterojunctions," Science, vol. 270, no. 5243, pp. 1789-1791, 1995.

[3] S. E. Shaheen, C. J. Brabec, N. S. Sariciftci, F. Padinger, T. Fromherz, and J. C. Hummelen, " $2.5 \%$ efficient organic plastic solar cells," Applied Physics Letters, vol. 78, no. 6, pp. 841-843, 2001.

[4] P. Schilinsky, C. Waldauf, and C. J. Brabec, "Recombination and loss analysis in polythiophene based bulk heterojunction photodetectors," Applied Physics Letters, vol. 81, no. 20, p. 3885, 2002.

[5] D. Veldman, S. C. J. Meskers, and R. A. J. Janssen, “The energy of charge-transfer states in electron donor-acceptor blends: insight into the energy losses in organic solar cells," Advanced Functional Materials, vol. 19, no. 12, pp. 1939-1948, 2009.

[6] S. Gunes, H. Neugebauer, and N. S. Sariciftci, "Conjugated polymer-based organic solar cells," Chemical Reviews, vol. 107, no. 4, pp. 1324-1338, 2007.

[7] B. C. Thompson and J. M. J. Fréchet, "Polymer-fullerene composite solar cells," Angewandte Chemie, International Edition, vol. 47, no. 1, pp. 58-77, 2008.

[8] A. J. Heeger, "Semiconducting polymers: the third generation," Chemical Society Reviews, vol. 39, no. 7, pp. 2354-2371, 2010.
[9] R. A. J. Janssen and J. Nelson, "Factors limiting device efficiency in organic photovoltaics," Advanced Materials, vol. 25, no. 13, pp. 1847-1858, 2013.

[10] S. H. Park, A. Roy, S. Beaupre et al., "Bulk heterojunction solar cells with internal quantum efficiency approaching 100\%," Nature Photonics, vol. 3, no. 5, pp. 297-302, 2009.

[11] J. Zhao, Y. Li, G. Yang et al., "Effcient organic solar cells processed from hydrocarbon solvents," Nature Energy, vol. 1, no. 2, pp. 15027-15034, 2016.

[12] B. Walker, A. B. Tamayo, X. D. Dang et al., "Nanoscale phase separation and high photovoltaic efficiency in solutionprocessed, small-molecule bulk heterojunction solar cells," Advanced Functional Materials, vol. 19, no. 19, pp. 30633069, 2009.

[13] W. Li, K. H. Hendriks, M. M. Wienk, and R. A. J. Janssen, "Diketopyrrolopyrrole polymers for organic solar cells," Accounts of Chemical Research, vol. 49, no. 1, pp. 78-85, 2016.

[14] J. C. Bijleveld, V. S. Gevaerts, D. Di Nuzzo et al., "Efficient solar cells based on an easily accessible diketopyrrolopyrrole polymer," Advanced Materials, vol. 22, no. 35, pp. E242E246, 2010.

[15] G. Dennler, M. C. Scharber, and C. J. Brabec, "Polymer-fullerene bulk-heterojunction solar cells," Advanced Materials, vol. 21, no. 13, pp. 1323-1338, 2009.

[16] A. Rao, P. C. Y. Chow, S. Gelinas et al., "The role of spin in the kinetic control of recombination in organic photovoltaics," Nature, vol. 500, no. 7463, pp. 435-439, 2013.

[17] S. D. Dimitrov, S. Wheeler, D. Niedzialek et al., "Polaron pair mediated triplet generation in polymer/fullerene blends," Nature Communications, vol. 6, p. 6501, 2015.

[18] I. A. Howard, N. C. Greenham, A. Abrusci, R. H. Friend, and S. Westenhoff, "Charge transfer and charge separation in conjugated polymer solar cells, chapter 13," in Nanostructured Conductive Polymers, A. Eftekhari, Ed., pp. 531-561, Wiley, 2010.

[19] M. B. Smith and J. Michl, "Singlet Fission," Chemical Reviews, vol. 110, no. 11, pp. 6891-6936, 2010.

[20] A. D. Becke, "Density-functional thermochemistry. III. The role of exact exchange," The Journal of Chemical Physics, vol. 98, no. 7, pp. 5648-5652, 1993.

[21] Y. Shao, L. F. Molnar, Y. Jung et al., "Advances in methods and algorithms in a modern quantum chemistry program package," Physical Chemistry Chemical Physics, vol. 8, no. 27, pp. 3172-3191, 2006.

[22] T. M. Clarke and J. R. Durrant, "Charge photogeneration in organic solar cells," Chemical Reviews, vol. 110, no. 11, pp. 6736-6767, 2010.

[23] I. H. M. van Stokkum, D. S. Larsen, and R. van Grondelle, "Global and target analysis of time-resolved spectra," Biochimica et Biophysica Acta, vol. 1657, no. 2-3, pp. 82-104, 2004.

[24] K. M. Mullen and I. H. M. van Stokkum, "TIMP: an R package for modeling multi-way spectroscopic measurements," Journal of Statistical Software, vol. 18, no. 3, pp. 1-46, 2007.

[25] J. J. Snellenburg, S. P. Laptenok, R. Seger, K. M. Mullen, and I. H. M. van Stokkum, "Glotaran: a Java-based graphical user interface for the R-package TIMP," Journal of Statistical Software, vol. 49, no. 3, pp. 1-22, 2012. October 2016, http://glotaran.org/.

[26] H. Lehtivuori, A. Efimov, H. Lemmetyinen, and N. V. Tkachenko, "Distributed decay kinetics of charge separated 
state in solid film," Chemical Physics Letters, vol. 437, no. 4, pp. 238-242, 2007.

[27] I. A. Howard, R. Mauer, M. Meister, and F. Laquai, "Effect of morphology on ultrafast free carrier generation in polythiophene:fullerene organic solar cells," Journal of the American Chemical Society, vol. 132, no. 42, pp. 14866-14876, 2010.

[28] A. A. Paraecattil, S. Beaupré, M. Leclerc, J.-E. Moser, and N. Banerji, "Intensity dependent femtosecond dynamics in a PBDTTPD-based solar cell material," Journal of Physical Chemistry Letters, vol. 3, no. 20, pp. 2952-2958, 2012.

[29] G. Grancini, N. Martino, M. R. Antognazza, M. Celebrano, H. J. Egelhaaf, and G. Lanzani, "Influence of blend composition on ultrafast charge generation and recombination dynamics in low band gap polymer-based organic photovoltaics," Journal of Physical Chemistry C, vol. 116, no. 17, pp. 98389844, 2012.

[30] I. A. Howard, J. M. Hodgkiss, X. P. Zhang et al., "Charge recombination and exciton annihilation reactions in conjugated polymer blends," Journal of the American Chemical Society, vol. 132, no. 1, pp. 328-335, 2010.

[31] T. Q. Nguyen, I. B. Martini, J. Liu, and B. J. Schwartz, "Controlling interchain interactions in conjugated polymers: the effects of chain morphology on exciton-exciton annihilation and aggregation in MEH-PPV films," The Journal of Physical Chemistry. B, vol. 104, no. 2, pp. 237-255, 2000.

[32] G. J. Denton, N. Tessler, M. A. Stevens, and R. H. Friend, "Optical response of conjugated polymers excited at high intensity," Synthetic Metals, vol. 102, no. 1-3, p. 1008, 1999.

[33] T. Ripolles-Sanchis, S. R. Raga, A. Guerrero et al., "Molecular electronic coupling controls charge recombination kinetics in organic solar cells of low bandgap diketopyrrolopyrrole, carbazole, and thiophene polymers," Journal of Physical Chemistry C, vol. 117, no. 17, pp. 8719-8726, 2013.

[34] C. G. Shuttle, B. O’Regan, A. M. Ballantyne, J. Nelson, D. D. C. Bradley, and J. R. Durrant, "Bimolecular recombination losses in polythiophene: fullerene solar cells," Physical Review B, vol. 78, no. 11, p. 113201, 2008.

[35] A. Pivrikas, G. Juscaronka, A. J. Mozer et al., "Bimolecular recombination coefficient as a sensitive testing parameter for low-mobility solar-cell materials," Physical Review Letters, vol. 94, no. 17, pp. 176806/1-176806/4, 2005.

[36] R. M. Williams, J. M. Zwier, and J. W. Verhoeven, "Photoinduced intramolecular electron transfer in a bridged $\mathrm{C}_{60}$ (acceptor)-aniline (donor) system; photophysical properties of the first "active" fullerene diad," Journal of the American Chemical Society, vol. 117, no. 14, pp. 40934099, 1995.

[37] P. A. Liddell, D. Kuciauskas, J. P. Sumida et al., "Photoinduced charge separation and recombination to a triplet state in a carotene-porphyrin-fullerene triad," Journal of the American Chemical Society, vol. 119, no. 6, pp. 1400-1405, 1997.

[38] M. R. Roest, A. M. Oliver, M. N. Paddon-Row, and J. W. Verhoeven, "Distance dependence of singlet and triplet charge recombination pathways in a series of rigid bichromophoric systems," The Journal of Physical Chemistry. A, vol. 101, no. 27, pp. 4867-4871, 1997.

[39] M. Niemi, N. V. Tkachenko, A. Efimov et al., "Exciplex mediated photoinduced electron transfer reactions of phthalocyanine-fullerene dyads," The Journal of Physical Chemistry. A, vol. 112, no. 30, pp. 6884-6892, 2008.
[40] T. Okada, I. Karaki, and N. Mataga, "Picosecond laser photolysis studies of hydrogen-atom transfer reaction via heteroexcimer state in pyrene-primary and pyrene-secondary aromatic amine systems: role of hydrogen-bonding interaction between amino group of donor and $\pi$ electrons of acceptor in the heteroexcimer," Journal of the American Chemical Society, vol. 104, no. 25, pp. 7191-7195, 1982.

[41] T. Okada, I. Karaki, E. Matsuzawa, N. Mataga, Y. Sakata, and S. Misumi, "Ultrafast intersystem crossing in some intramolecular heteroexcimers," The Journal of Physical Chemistry, vol. 85, no. 26, pp. 3957-3960, 1981.

[42] B. P. Karsten, R. K. M. Bouwer, J. C. Hummelen, R. M. Williams, and R. A. J. Janssen, "Charge separation and (triplet) recombination in diketopyrrolopyrrole-fullerene triads," Photochemical \& Photobiological Sciences, vol. 9, no. 7, pp. 1055-1065, 2010.

[43] P. W. M. Blom, V. D. Mihailetchi, L. J. A. Koster, and D. E. Markov, "Device physics of polymer:fullerene bulk heterojunction solar cells," Advanced Materials, vol. 19, no. 12, pp. 1551-1566, 2007.

[44] G. Juska, K. Genevicius, R. Osterbacka et al., "Initial transport of photogenerated charge carriers in pi-conjugated polymers," Physical Review B, vol. 67, no. 8, p. 081201, 2003.

[45] Y. S. Cho and R. R. Franklin, "Conducting polymer material characterization using high frequency planar transmission line measurement," Transactions on Electrical and Electronic Materials, vol. 13, no. 5, pp. 237-240, 2012.

[46] R. M. Williams, N. Van Anh, and I. H. M. van Stokkum, “Triplet formation by charge recombination in thin film blends of perylene red and pyrene: developing a target model for the photophysics of organic photovoltaic materials," The Journal of Physical Chemistry. B, vol. 117, no. 38, pp. 11239-11248, 2013.

[47] S. Gélinas, A. Rao, A. Kumar et al., "Ultrafast long-range charge separation in organic semiconductor photovoltaic diodes," Science, vol. 343, no. 6170, pp. 512-516, 2014.

[48] B. Hu, L. Yan, and M. Shao, "Magnetic-field effects in organic semiconducting materials and devices," Advanced Materials, vol. 21, no. 14-15, pp. 1500-1516, 2009.

[49] D. N. Congreve, J. Lee, N. J. Thompson et al., "External quantum efficiency above $100 \%$ in a singlet-exciton-fission based organic photovoltaic cell," Science, vol. 340, no. 6130, pp. 334-337, 2013.

[50] J. Guo, H. Ohkita, H. Benten, and S. Ito, "Near-IR femtosecond transient absorption spectroscopy of ultrafast polaron and triplet exciton formation in polythiophene films with different regioregularities," Journal of the American Chemical Society, vol. 131, no. 46, pp. 16869-16880, 2009.

[51] J. W. Verhoeven, "On the role of spin correlation in the formation, decay, and detection of long-lived, intramolecular charge-transfer states," Journal of Photochemistry and Photobiology, C: Photochemistry Reviews, vol. 7, no. 1, pp. 40-60, 2006.

[52] M. T. Colvin, A. B. Ricks, A. M. Scott, D. T. Co, and M. R. Wasielewski, "Intersystem crossing involving strongly spin exchange-coupled radical ion pairs in donor-bridge-acceptor molecules," The Journal of Physical Chemistry. A, vol. 116, no. 8, pp. 1923-1930, 2012.

[53] K. M. Lefler, K. E. Brown, W. A. Salamant, S. M. Dyar, K. E. Knowles, and M. R. Wasielewski, "Triplet state formation in 
photoexcited slip-stacked perylene-3,4:9,10-bis(dicarboximide) dimers on a xanthene scaffold," The Journal of Physical Chemistry. A, vol. 117, no. 40, pp. 10333-10345, 2013.

[54] M. A. El-Sayed, "Effect of spin orbit interactions on the dipolar nature of the radiative microwave zero-field transitions in aromatic molecules," The Journal of Chemical Physics, vol. 60 , no. 11 , pp. $4502-4507,1974$.

[55] Z. E. X. Dance, Q. Mi, D. W. McCamant, M. J. Ahrens, M. A. Ratner, and M. R. Wasielewski, "Time-resolved EPR studies of photogenerated radical ion pairs separated by p-phenylene oligomers and of triplet states resulting from charge recombination," The Journal of Physical Chemistry. B, vol. 110, no. 50, pp. 25163-25173, 2006.

[56] Z. E. X. Dance, S. M. Mickley, T. M. Wilson et al., "Intersystem crossing mediated by photoinduced intramolecular charge transfer: julolidine-anthracene molecules with perpendicular $\pi$ systems," The Journal of Physical Chemistry. A, vol. 112, no. 18, pp. 4194-4201, 2008.

[57] M. T. Colvin, A. B. Ricks, A. M. Scott et al., "Magnetic fieldinduced switching of the radical-pair intersystem crossing mechanism in a donor-bridge-acceptor molecule for artificial photosynthesis," Journal of the American Chemical Society, vol. 133, no. 5, pp. 1240-1243, 2011. 

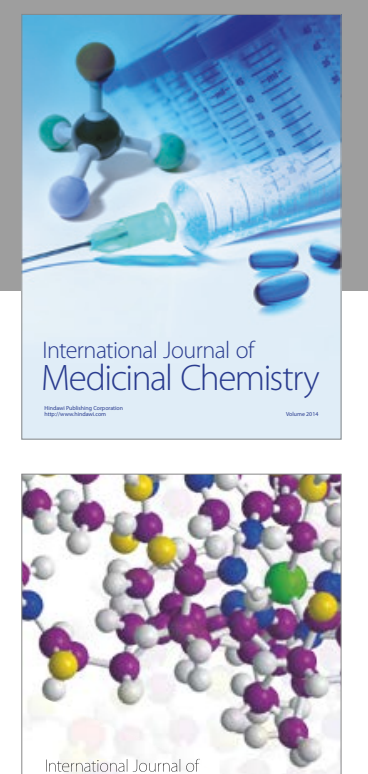

Carbohydrate Chemistry

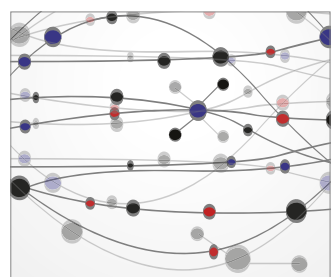

The Scientific World Journal
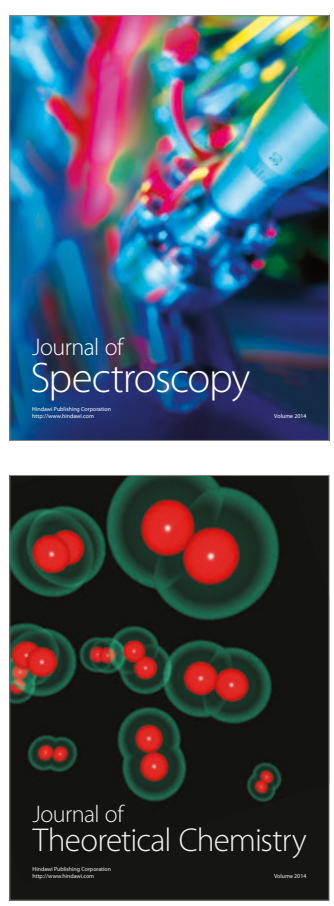
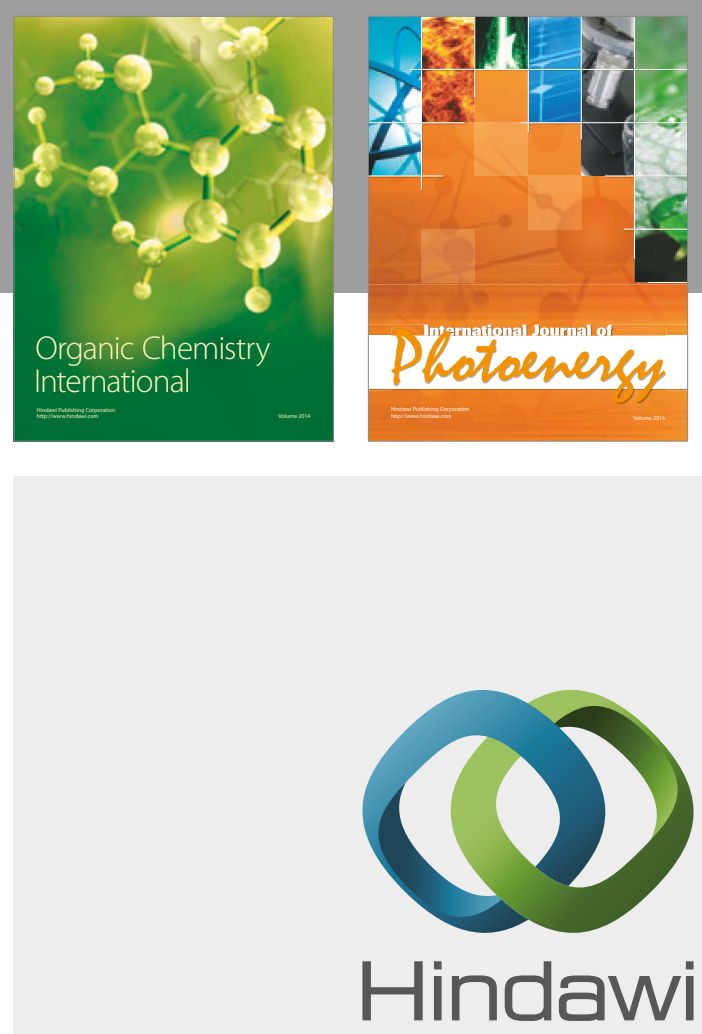

Submit your manuscripts at

https://www.hindawi.com

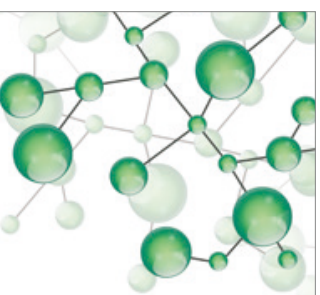

International Journal of

Inorganic Chemistry

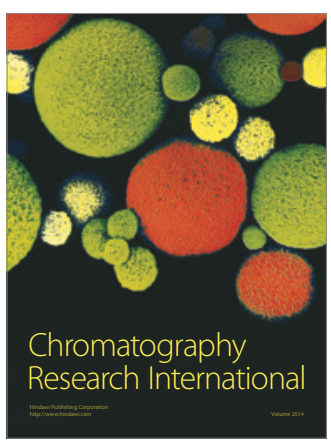

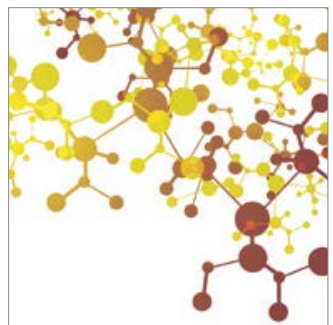

Applied Chemistry
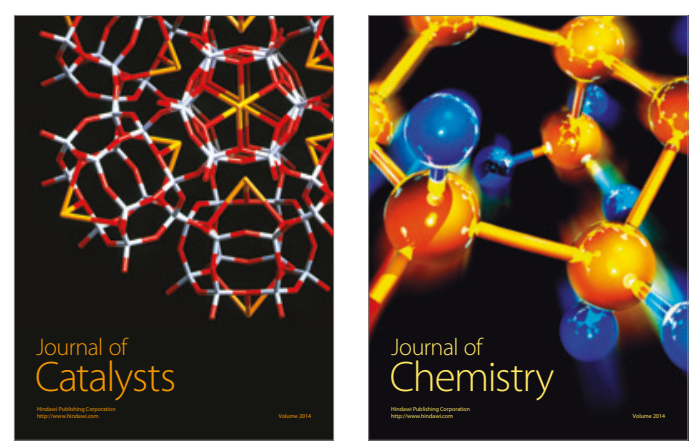
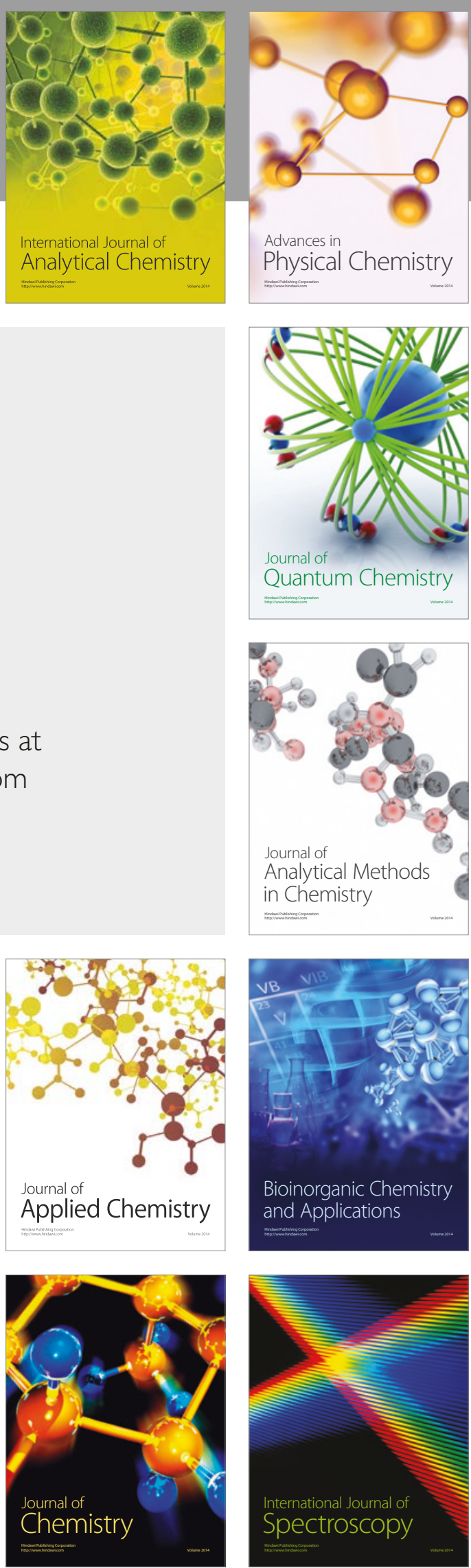